\title{
Predicting mind wandering episodes quarter hours in advance s from EEG
}

\author{
Chie Nakatani $^{1, *}$, Hannah Bernhard ${ }^{1,2}, \&$ Cees van Leeuwen ${ }^{1,3}$
}

Affiliations:

1: Brain and Cognition Research Unit, KU Leuven, Tiensestraat 102 - Box 3711, 3000 Leuven, Belgium

2: Department of Cognitive Neuroscience, Faculty of Psychology and Neuroscience, Maastricht University, Maastricht, The Netherlands

3: Center for Cognitive Science, TU Kaiserslautern, Kaiserslautern, Germany

*Corresponding author: Chie Nakatani Email: chie.nakatani@ kuleuven.be

Authors’ Email: chie.nakatani@kuleuven.be, hannah.bernhard@maastrichtuniversity.nl, cees.vanleeuwen@kuleuven.be

Key words: Spontaneous mental process, cognitive control, default mode network, dynamical model, delay, attention, working memory, alpha amplitude, evoked potentials

Funding: This work was supported by an Odysseus grant from The Flemish Organization for Science PEP-C3738-G.0003.12 to CvL 


\begin{abstract}
Mind wandering occurs when spontaneous thought generation is increased while cognitive control is decreased, presumably in response to antagonistic effects of default mode- (DMN) and goal-directed network (GDN) activity. Previous studies assumed that DMN or GDN activity manifests in behavior with minimal delays and has rigid effects, i.e., DMN always facilitates generation of spontaneous thought and inhibits cognitive control. With those static response functions, the antagonism of DMN and GDN could explain the alternation of task focused and mind wandering states. But it rules out others, such as multitasking, where spontaneous thought and cognitive control are both increased, or rumination, where both are decreased. However, shifts from task focused to mind wandering have been shown to occur via a multi-tasking state. We therefore propose dynamic response functions, allowing delay or reversal of DMN and GDN effects. Dynamic response functions enabled us to stochastically predict mind wandering up to at least $30 \mathrm{~min}$ in advance from EEG measures of DMN and GDN activity in a tone counting task. Experimental results show both considerable delays and switches between task-positive and negative effects. Mind wandering was initiated by a barrage of DMN activity more than 20 minutes prior a report of mind wandering, directing the mental focus inward. Critically, facilitation of spontaneous thoughts occurred several minutes prior to the report. These thoughts sustained up to $10 \mathrm{~min}$, after which a rebound to task-focused behavior was effectuated, notably without intervention from the GDN. Repeated GDN interventions, however, secured a basic level of task performance.
\end{abstract}

\title{
250 words
}




\section{Introduction}

The wakeful mind continuously generates thoughts, including ones irrelevant to the present situation. Such thoughts can distract us and lead us into an episode of mind wandering (Smallwood \& Schooler, 2006). The more of these thoughts come up, the more possibilities to lose focus (Mason et al., 2007; Nakatani, Ganschow, \& van Leeuwen, 2019). Yet, merely having many thoughts in mind does not always lead to mind wandering. The functions believed to prevent this are collectively known as cognitive control, i.e., those functions that focus thought to make willful and goal-directed behavior possible (Aben, Verguts, \& Van den Bussche, 2017; Cohen, Dunbar, \& McClelland, 1990; Funes, Lupiáñez, \& Humphreys, 2010; Otto, Skatova, Madlon-Kay, \& Daw, 2015; Ridderinkhof, 2002). Even if numbers of emerging thoughts are large, the task-relevant ones will prevail as long as cognitive control is up. A mind wandering episode is therefore most likely to arise while thought generation is high but cognitive control subsides (Nakatani et al., 2019).

As the levels of spontaneous thought and cognitive control change, episodes of mind wandering and focused states may alternate. This alternation could be understood as a function of brain dynamics. As an oscillatory phenomenon, it is likely an effect of the antagonism of two neural systems. One is the Default Mode Network (DMN). This system encompasses widespread brain regions, including prefrontal cortex, posterior cingulate cortex, as well as precuneus and memory-related regions such as the parahippocampal and middle temporal gyri (Raichle, 2015; Raichle et al., 2001). DMN regions show higher activity in resting conditions than during task performance. Such task-negativity was observed for a variety of neural measures, including the ratio of oxygen consumption to blood flow in PET (Raichle et al., 2001), BOLD in fMRI (Greicius, Krasnow, Reiss, \& Menon, 2003), intercranial (stereo) EEG (Jerbi et al., 2010), source-estimated scalp EEG (Laufs et al., 2003; Mantini, Perrucci, Del Gratta, Romani, \& Corbetta, 2007) and MEG (Brookes et al., 2011). DMN regions generally show higher activation during mind wandering than in task-focused conditions, both in BOLD (Christoff, Gordon, Smallwood, Smith, \& Schooler, 2009; Fox, Spreng, Ellamil, Andrews-Hanna, \& Christoff, 2015; Mason et al., 2007) and in 
M/EEG measures such as amplitude of alpha band activity (Chen, Feng, Zhao, Yin, \& Wang, 2008; Compton, Gearinger, \& Wild, 2019; Gruberger, Simon, Levkovitz, Zangen, \& Hendler, 2011; Marino, Arcara, Porcaro, \& Mantini, 2019).

The second system consists of the set of task-positive networks. This system encompasses the dorsal attention network, including the dorsolateral prefrontal cortex and the intraparietal sulcus (Corbetta, Kincade, Ollinger, McAvoy, \& Shulman, 2000; Hopfinger, Buonocore, \& Mangun, 2000), the salience network, including the insula and anterior cingulate cortex (Menon \& Uddin, 2010; Seeley et al., 2007), and the sensory-motor systems. Increased activity in these subsystems characterizes various forms of goaldirected behavior (Cole \& Schneider, 2007). As a collective, we will call them the Goal-Directed Networks (GDN).

DMN and GDN appear to be antagonists. Overall, GDN activity anticorrelates with that of DMN (Fox et al., 2005; Fransson, 2005) Supposedly, then, DMN activity facilitates spontaneous thought generation while suppressing cognitive control whereas, vice versa, GDN activity facilitates cognitive control while suppressing spontaneous thought generation. In this way, the push and pull of DMN and GDN could modulate psychological functions, and lets them oscillate in an anticorrelated fashion. As one system temporarily takes the upper hand over the other, episodes of mind wandering proneness alternate with more focused ones (Figure 1a).

However, recent empirical studies suggest systematic deviations from anticorrelation. In Nakatani et al. (2019), experience sampling techniques were used to collect mind wandering reports during the wakeful hours over periods of 14 days. Participants concurrently reported the quantity and experiential content of spontaneous thoughts, and the level of cognitive control was measured by a simple online task. Results were plotted as trajectories in a 2-dimensional space, with the dimensions spontaneous thought and cognitive control (Figure 1b). Regions in this space denote a variety of mental states, for which DMN and GDN activity have been considered as major neural contributors (Christoff, Irving, Fox, Spreng, \& Andrews-Hanna, 2016; Voss \& Voss, 2014). As expected, mind wandering occurred in the region where 
thought production is high and cognitive control is low. However, the trajectory leading to that state was far more complex than what a simple antagonism of DMN and GDN could provide. It would have permitted only those states existing near the antidiagonal of Figure 1b. Yet, in particular before entering the mind wandering state, the trajectory typically visits regions in which cognitive control and thought production are both high. Mental states in such regions might, for instance, reflect multi-tasking behavior and were independently postulated for creative thinking (Fink, Graif, \& Neubauer, 2009; Jaarsveld \& Van Leeuwen, 2005). The very occurrence of such states suggests that the strict antagonism of DMN and GDN needs to be relaxed.

One breach of the antagonism seems to occur, in particular, when the task focus changes from externally, e.g., sensory stimulation, to internally focused, e.g., autobiographical, thoughts. Some DMN regions then change from task-negative to positive (Andrews-Hanna, Saxe, \& Yarkoni, 2014). The same could happen in the transition to mind wandering. Graded shifts are possible as well, as DMN contains subregions of which the response to a task differ from each other (Buckner \& Dinicola, 2019 for a recent review).

Besides the direction of the effect, another possibly relevant variable is delay. There may be a considerable latency, or time lag, between the onsets of a neural activity and the time a psychological function becomes manifested in behavior. Little is known about the full extent and variation of these lags. Previous studies have predominantly studied short delays; milliseconds to seconds in EEG, (e.g., 12s EEG segments before the subjective report of a mental state, Kirschner, Kam, Handy, \& Ward, 2012), In fMRI, lags may encompass seconds to a minute,(e.g., the hemodynamic response latency to an external stimulus in Boynton, Engel, Glover, \& Heeger, 1996). These studies could have missed more remote effects of neural activity, beyond the range of an event-response cycle. Much slower cycles were observed in Nakatani et al. (2019). Participants spent on average 10 min of mind wandering before it was reported in an experience sampling query. The critical neural activity could thus have occurred anywhere during this 10 min interval, or even earlier. 
The previous studies used mental states, e.g., self-report of mind wandering, or a behavioral measure of the state such as the error rate of an experimental task, to predict neural activity. In the current approach, neural activity is the predictor of the psychological functions. Proper assignment of model terms (neural predictor and psychological response variables) is critical for understanding dynamic neural effects. Moreover, the previous formulation does not distinguish mind wandering as a mental state in a state space like Figure 1b, and the levels of thought generation and cognitive control, which are the constitutive dimensions of the state space. The mental state and its dimensions can be measured independently, by using a combination of self report and task performance measures.

To take into account the above considerations, the relationship between neural activity and psychological functions may be described by a dynamical response function $\mathrm{F}_{\mathrm{N}, \Psi, t}(\delta \mathrm{t})$, where $\mathrm{N}$ is a neural system, $\{\mathrm{DMN}, \mathrm{GDN}\}$ and $\Psi$ the psychological response of interest thought generation, cognitive control $\}, t$ is time, and $\delta \mathrm{t}$ is time lag (Figure $1 \mathrm{c}$, the current model). Instead of a fixed direction of effect (always positive for DMN effects on spontaneous thought generation and GDN effects on cognitive control, and always negative for DMN effects on cognitive control and GDN effects on spontaneous thought generation) and a fixed and short delay (within reach of the classical event-response cycle, $<1$ $\min$ ), the response function can have a positive or negative effect, as well as large delays. The function may differ between focused and mind wandering states which are also labeled by $t$.

Our aim is to obtain empirical correlates of $\mathrm{F}_{\mathrm{N}, \Psi, \mathrm{t}}(\delta \mathrm{t})$, viz. $\widehat{F}_{N, \Psi, S}(\Delta t)$, at discrete lags $\Delta t$ (as the data are sampled over discrete intervals) for $N=\{$ DMN-related activity, GDN-related activity $\}$ and $\Psi=$ \{self-reported thought generation, behavioral measure of cognitive control $\}$ in mental states, $S=\{$ mind wandering, task focused \}. Subscript $S$ is used instead of t to distinguish state-specific effects from general effects of time on task, e.g., fatigue. In each $\hat{F}$, we will identify at which lags $\Delta t, N$ makes a significant contribution to $\Psi$, and whether the direction of the effect is positive or negative. To obtain DMN- and GDN-related activities, we recorded EEG during a simple and repetitive, mind-wandering inducing task. As a measure of thought generation, we used the self-reported number of thoughts whereas task 
performance is taken as a measure of cognitive control. Independent from these measures, rating of mind wandering level was also repeated during the task. Timings of the high- and low level of mind wandering rating were used as time reference points for the mind wandering and task focused states during the task session. The results show neural effects switching in direction and operating with long, mental-state specific delays, navigating the mind between task focused and mind wandering states. 
bioRxiv preprint doi: https://doi.org/10.1101/2021.09.04.458977; this version posted September 6, 2021. The copyright holder for this preprint (which was not certified by peer review) is the author/funder, who has granted bioRxiv a license to display the preprint in perpetuity. It is made available under aCC-BY-NC-ND 4.0 International license.

a)

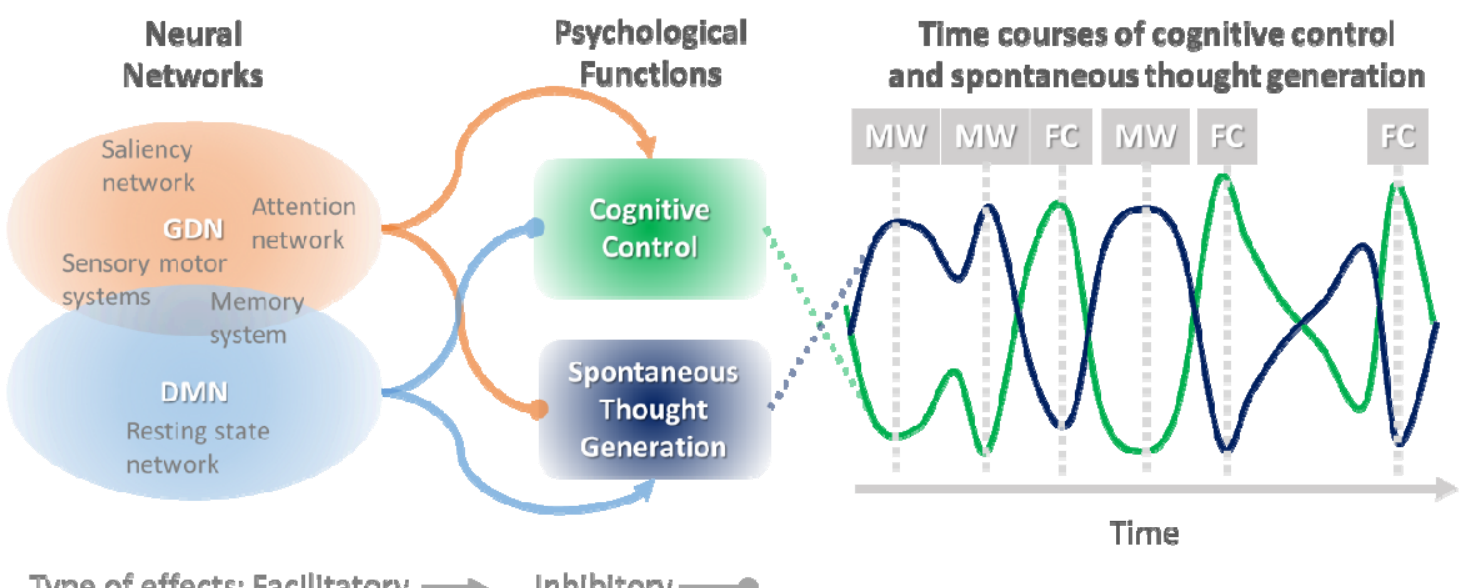

b)
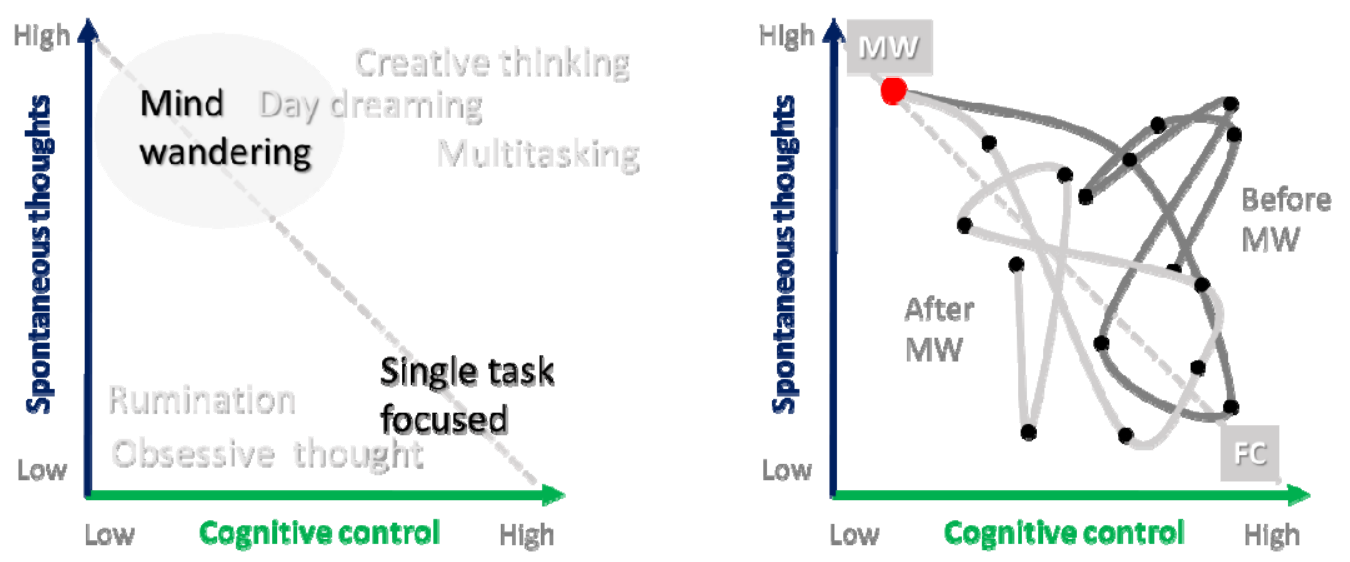

c)

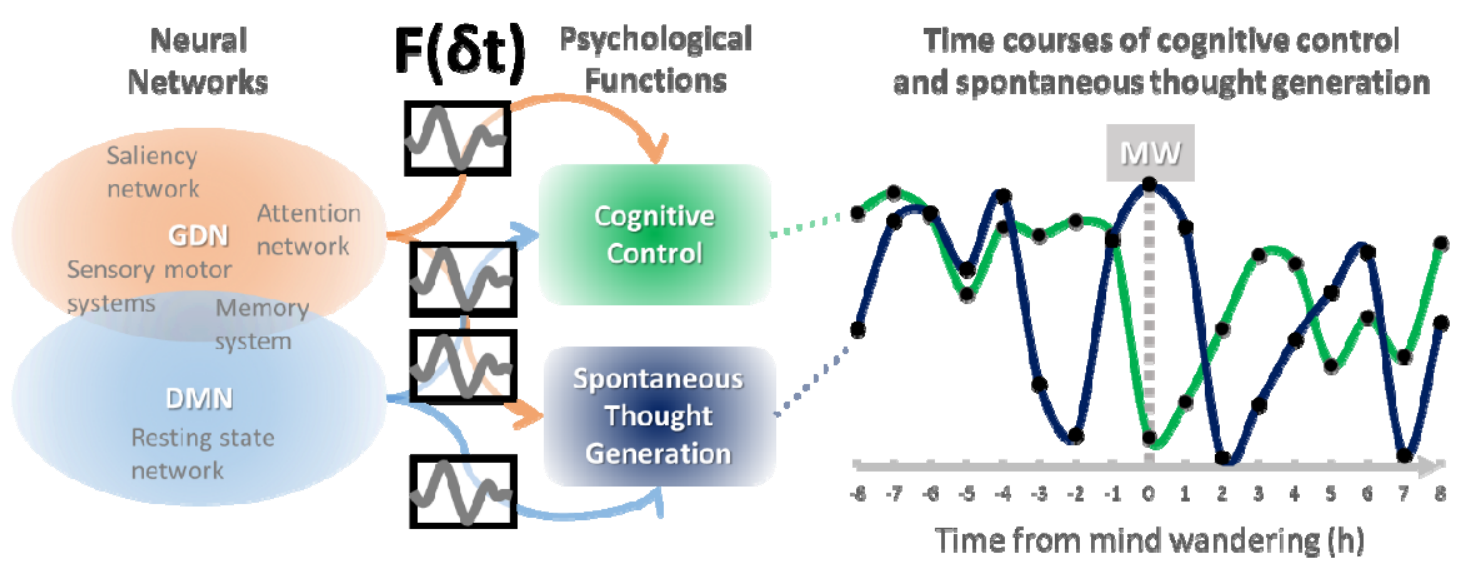


Figure 1. The classical neurodynamic model, the state space, and the current model for mind wandering and related mental states.

a) In a classical model of goal-directed behavior, goal-directed networks (GDN) collectively facilitate cognitive control, while suppressing spontaneous thought generation. Networks collectively known as the default mode network (DMN) facilitate thought generation, while suppressing cognitive control. The model predicts that around a report of mind wandering (MW) cognitive control (green line) is low and thought generation (dark blue line) is high, while the opposite is the case when a task-focused state (FC) is reported. However, the model fails to take into account possible switches from task-negative to taskpositive in the DMN as well as variable time lags between neural activity and behavioral effects.

b) Left panel: Mind wandering and single task focused behavior in a state space defined by the dimensions of spontaneous thought generation and cognitive control. According to the classical model, transitions from the task focused to the mind wandering state, and vice versa, would gravitate along the antidiagonal (dotted grey line). Right panel: empirical trajectory before (dark gray, 8 hours) and after (light gray, 8 hours) a report of mind wandering (Nakatani et al., 2019). Note the complexity of the trajectory and the divergence from the antidiagonal.

c) The current model considers the DMN and GDN effects on each psychological function as a dynamical response function, $\mathrm{F}(\delta \mathrm{t})$ which is assumed as context dependent (additional explanation in the text). 


\section{Methods}

\section{Overview}

Participants performed the task of counting tones up to a target number pre-specified at the start of the trial. Each time after reaching the target, they retrospectively rated their mental states during the trial on a scale, ranging from fully task focused to fully mind wandering (\%MW), and provided a subjective report of the number of thoughts (NoTs) that had occurred during the trial. NoTs were used as measure of spontaneous thought generation; accuracy of the tone count response ('correct count', or CC), was used as behavioral measure of cognitive control. EEG was recorded continuously during the task. As the neural measure of GDN activity, amplitude of the auditory evoked potential (AEP) to the tone stimuli was used. AEP amplitude correlates positively with auditory attention, for which the GDN is held responsible (Picton \& Hillyard, 1974; Picton, Hillyard, Krausz, \& Galambos, 1974). As the neural measure of DMN activity, amplitude of alpha band activity $(8-12 \mathrm{~Hz})$ was used. Alpha amplitude is the primary EEG measure of DMN activity (Lehtelä, Salmelin, \& Hari, 1997). Crucial to the current study, using alpha band activity avoids overlap with the main band of the AEP which is situated in the theta band $(4-8 \mathrm{~Hz}$, Bernhard, 2018). In sum, a sequence of trials thus provided 5 parallel time series, one each for \%MW, NoTs, CC, alpha, and AEP amplitude (Figure 2).

The time series were analyzed relative to the time points of high $\% \mathrm{MW}$ reports $(\geqq 70 \%)$ and those of low $\%$ MW reports $(<30 \%)$. For both reference time points, event time-locked averaging was conducted for the NoTs, CC, alpha, and AEP time series, respectively. A set of general linear mixed models (GLMM) was applied to the averaged time series to estimate the sign (positive or negative) and time lag of the neural effects up to 30 min prior to the \%MW report. This yielded series of $\hat{F}(\Delta t)$ values for the mind wandering and task focused states. The results show in what lag and with which sign, a neural effect occurred in each of the 4 combinations of $\mathrm{N}$ and $\Psi$ (Figure 2b). Expected results are described in the section Estimation of direction and timing of the neural effects. 
bioRxiv preprint doi: https://doi.org/10.1101/2021.09.04.458977· this version posted September 6, 2021. The copyright holder for this preprint (which was not certified by peer review) is the author/funder, who has granted bioRxiv a license to display the preprint in perpetuity. It is made available under aCC-BY-NC-ND 4.0 International license.

a)

Tone Counting task

(ग)) \|\|\|\|\|\|\|\|\|\|\|\|\|\|$\|$ Tone: $440 \mathrm{~Hz} / 100 \mathrm{~ms}$ SOA $2000-2500 \mathrm{~ms}$ "Count N tones" (n: 20-24)

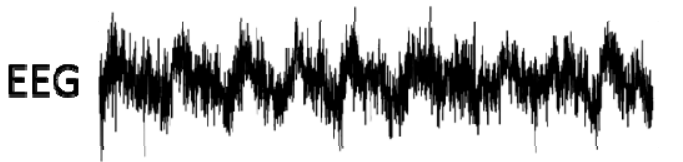

$\sim 2 \mathrm{~min} /$ trial

b)
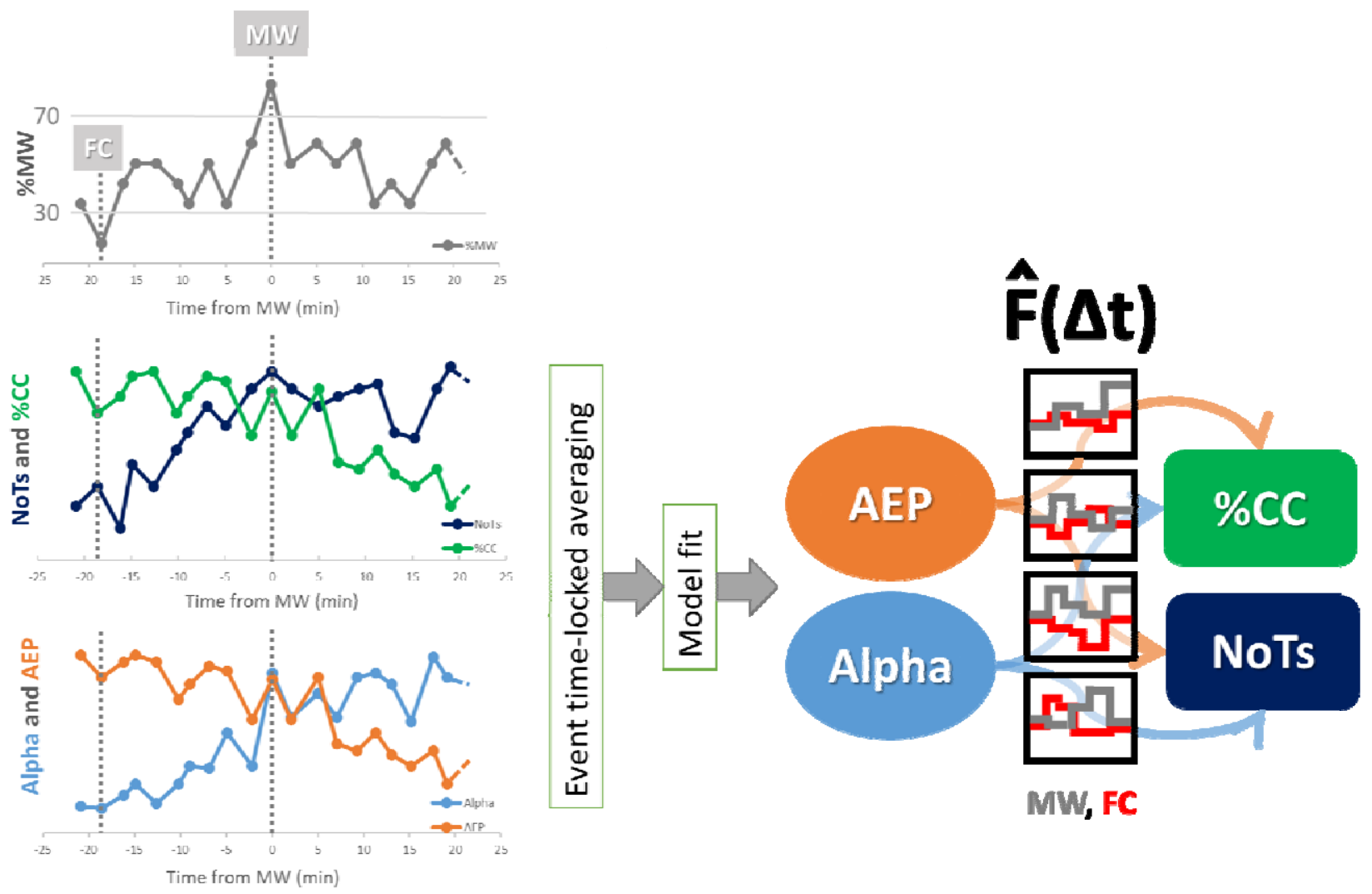
Figure 2. Illustration of the methods

a) Participants counted up to n (20-24) tones, and pressed a key when the count was reached. After the key press, they reported the percentage of time spent for mind wandering $(\% \mathrm{MW})$ and the number of thoughts (NoTs) during the trial. EEG was recorded during the task. Amplitude of alpha activity and auditory evoked response (AEP) were computed from the EEG. Therefore, each trial yields 5 measures: Correctness of the count (CC), NoTs, \%MW, Alpha and AEP amplitude.

b) The line graphs illustrate time series of the measures. The time series were analyzed from the time point of a high $\% \mathrm{MW}(\geqq 70 \%)$, or a low $\% \mathrm{MW}(<30 \%)$ report (thus task-focused: FC). First, the time series of NoTs, \%CC, Alpha, and AEP were averaged in event-time-locked manner, i.e., each of the time series was segmented, aligned, and averaged from the high- and low \%MW report, respectively. Next, mixed-effect models were fit to the averaged data to estimate the neural effects on the psychological measures, $\hat{F}(\Delta t)$. The results show the direction and the timing of the neural effects (more explanation in the text). 


\section{Ethical approval and Participants}

The study was approved by The KU Leuven Medical Ethics committee (protocol number: S56128). Twenty healthy volunteers (average 23.00 years, 6 male) participated in exchange for monetary compensation (10€/hour). They were recruited via the online participant recruitment system at KU Leuven. Prior to the participation, they were given information about the study and gave oral and written informed consent. All data were collected prior to the onset of the 2020 COVID19 pandemic.

\section{Transparency and Openness}

All data are at the project page on Open Science Framework (https://osf.io/5ucvs/). They will be made publicly available after publication of the manuscript.

\section{Tasks}

The tone counting task (Figure 2a) was used to elicit a sizeable incidence rate of mind wandering during an approx. 2-hour period. The task consisted of 37 trials lasting approx. 90s each, in which a sequence of brief tones, $100 \mathrm{~ms}$ in $440 \mathrm{~Hz}$ frequency, was presented at $70 \mathrm{~dB}$ via the speakers located at right and left sides of a monitor in front of them. The tones were presented with a stimulus onset asynchrony (SOA) between 2000 and 2500ms (pseudo-randomized with 100ms steps). At the beginning of each trial, a number was presented in the center of the display. The number indicated how many tones had to be counted before a response. The number of tones to be counted ranged between 20 and 24 (pseudorandomized over trials). Participants put the right index finger on Key " 5 " of the ten-key pad and closed their eyes before the series of tones started. When the target number was reached, participants responded by pressing the key. Then they opened their eyes and reported the percentage of time spent on task irrelevant thoughts in 0 to $100 \%$ in $10 \%$ steps. An eleven-point scale $(0 \%--10 \%--. . .-90 \%--100 \%)$ was shown on the display and participants reported the percentage (\%MW) using the ten-key pad. Lastly, participants reported on the keypad the number of thoughts (NoTs) that had occurred in their minds during the trial on a ten-point scale. The scale ranged from 1: only task-related thought to 10: task-related and 9 
or more task-irrelevant thoughts. Participants were instructed to evaluate the number of thoughts in a consistent manner over trials. For instance, they were instructed to report "1" when they had only taskrelated thoughts; when they had one task-irrelevant thought, for instance about having lunch after the experiment, they reported "2". They were told that items included in the thoughts (e.g., a sandwich, an apple, and a bottle of soda) were not counted separately. If two different thoughts occurred at different times during the trial (e.g., thoughts on lunch, then later during the trial thinking about doing laundry), they reported "3." And so on. The instruction was the same as that in Nakatani et al., (2019). Participants had the opportunity to take a break at marked sections of the task. EEG was recorded during the task. (Cf. Data Analysis for more details of EEG measures)

An Auditory Oddball Task was applied prior to the tone-counting task to specify the relevant parameters of the auditory evoked potentials (AEP), namely, scalp distribution, latency, and main frequency band, for subsequent use in the tone counting task. Participants placed their right index finger on the " 5 " key of the ten-key pad and closed their eyes. Then, a series of 110 tones was presented at $70 \mathrm{~dB}$ via the speakers, with an SOA pseudo-randomized between 2000 and $2500 \mathrm{~ms}$ in $100 \mathrm{~ms}$ steps. Of these tones, 101 were standard $(440 \mathrm{~Hz}, 100 \mathrm{~ms}$ duration), physically identical to the ones used in the tone counting task, and 5 were odd-ball tones $(510 \mathrm{~Hz}, 100 \mathrm{~ms}$ duration), which were randomly interspersed. Participants were asked to respond to the oddballs by pressing the key as fast as possible.

Both tasks were implemented using PsychoPy Version 1.82.01 (Peirce, 2009) on Windows XP.

\section{EEG recording}

EEG was recorded using the Patientguard EEG recording system (ANT Neuro, Enschede, The Netherlands). Of 64 channels in total, 59 were EEG channels and the remaining ones were used for recording the electrooculogram (EOG, vertical and horizontal) and recording reference (right mastoid, was later re-referenced to average reference). The placement of the EEG electrodes was according to the 
manufacturer-defined whole head system rather than the $10-10$ system. The signal was sampled at $2048 \mathrm{~Hz}$. Electrode impedance was $20 \mathrm{kOhm}$ or less (for the majority, the impedance was $5 \mathrm{kOhm}$ or less).

\section{Procedure}

Experiments were conducted with participants individually in an EEG-laboratory of the University Hospital of Leuven. After electrodes were attached, the participant was seated in a dimly lit room in front of speakers and a monitor. Baseline EEG was recorded (eyes open/relax $1 \mathrm{~min}$, and eyes close/relax, 1 min). This was followed by an auditory oddball task for estimation of evoked response parameters, and next by the tone counting task. EEG was recorded during both tasks. Afterwards, baseline EEG measurement was performed again. Then the electrodes were removed, and the participant washed off the gel before leaving. The experimental session took about 3 hours in total.

\section{Data analysis}

\section{Labeling of tone counting trials}

Each tone counting trial was labeled based on the \%MW as a high, intermediate-level, or low mind wandering trial as follows. A histogram was compiled from all \%MW reports (Appendix, Figure A1). Based on the histogram, trials with $\% \mathrm{MW} \geqq 70$ were labeled as high mind wandering trials (on average, 9.11 trials). Trials with $40 \leqq \% \mathrm{MW}<60$ were labeled as intermediate-level mind wandering trials (on average, 10.58 trials). Trials with $\% \mathrm{MW}<30$ were labeled as low mind wandering trials (on average, 6.53 trials). As explained in the next section, the labels were used for event time-locked averaging of time series data.

\section{Preprocessing for time series of trial data}

The tone counting task was self-paced. The inter-trial interval was approx. 2 min, considering the time stamp of a \%MW report as the time stamp of the trial and all measures of the trial. From the time series of the trials, the time course of the neural and psychological measures over the trials around high, low, and medium \% MW reports were extracted using event time-locked averaging. For the high \% MW reports, 
first, all trials in time series were re-timed relative to the time stamp of a high $\% \mathrm{MW}$ report as Time 0. This yielded $\mathrm{m}$ re-timed time series per participant, where $\mathrm{m}$ is the number of the high \%MW report. The $\mathrm{m}$ time series were aligned to each other from Time 0 (Cf. Appendix Fig. A2). Second, data points of the aligned time series were collated in 2-min intervals prior to the averaging to accommodate differences in inter-trial intervals. E.g., the data points between Time 0 and $1 \mathrm{~min} 59.99 \mathrm{sec}$ were collated in Bin 0. Each bin numbers correspond to the starting time of the bin, e.g., Bins 2, 4, and 6 correspond to bins which start 2, 4, and 6 min after Time 0, respectively; Bins -2, -4, and -6 start 2, 4, and 6 min before Time 0, respectively. Third, a bin average was computed for each measure; CC, NoTs, AEP and alpha amplitude (details of these EEG measures are explained in Computation of AEP and DMN-related alpha amplitude). Averaged CC was multiplied by 100 to obtain \%CC. The event time-locked averaging procedure yielded interval series of \%CC, NoTs, AEP, and alpha amplitude per participant, approximately between Bins-70 and 70 over all participants. The number of data points for the averaging decreased for the bins far from Time 0 . In the following analyses, we used data segments of which number of data was sufficient for the general linear mixed model analysis (which is robust against uneven number of samples, (Pinheiro \& Bates, 2006)). More details of the interval series data, including the number of data per time bin, are reported in Appendix.

For the low and intermediate \%MW reports, two more interval series were compiled likewise; one with respect to the time stamp of the low $\% \mathrm{MW}$ report and the other with respect to the intermediatelevel \%MW report. The interval series for the high, low, and intermediate \%MW reports showed different temporal patterns. An analogy to average Event Related Potentials (ERPs) might be helpful here; ERPs obtained from different event markers of the same EEG data show different waveforms. The interval series relative to the high and low $\% \mathrm{MW}$ report were used in the main data analysis, while those from the mid-level \%MW report were used to identify DMN-related alpha activity.

\section{EEG Preprocessing}


EEG data were pre-processed using Brain Vision Analyzer (version 2.1.2, Brain Products GmbH, Germany) and in-house Python scripts. Data were down-sampled from 2048 to $1024 \mathrm{~Hz}$, re-referenced to the average of all EEG channels, and filtered between 0.32 and $45.00 \mathrm{~Hz}$ using a $2^{\text {nd }}$ order zero phase shift Butterworth filter. Independent component analysis (ICA, Infomax algorithm) was applied to the filtered data for artifact removal. EOG channels were included in the ICA analysis. Ocular, cardiac, and muscular artifact components were visually identified and excluded from the inverse ICA.

To obtain alpha-band amplitude, the cleaned signals were band-passed between 8 and $12 \mathrm{~Hz}$. Hilbert transform was applied, and instantaneous amplitude was computed from the analytic signal. The amplitude signal was normalized per participant, using mean and standard deviation of a $2 \mathrm{~s}$ of noise-free sample from the pre-task baseline period. The normalized alpha amplitude was then segmented between 0 and 2000ms from the tone onset. Amplitude was averaged over a segment and further over a trial (20 to 24 segments per trial). This average was used as the alpha amplitude of the trial.

\section{Computation of AEP and DMN-related alpha amplitude}

Scalp distribution, peak latency, and peak frequency band of AEP were identified using the EEG data of the standard tones in the odd-ball task. The data were segmented from the onsets of the standard tones, between -500 and $+1500 \mathrm{~ms}$. The complex-Morlet wavelet transform was applied to the segment. Amplitude was computed from the complex-valued analytic signal. The amplitude in the time-frequency domain was normalized and averaged for each participant. The t-statistic and accompanying probability were computed for each time-frequency point. Type-I error was corrected using the false discovery rate (FDR) method (Benjamini \& Hochberg, 1995). FDR was applied to the p-values of all data points over the 59 EEG electrodes. Amplitude in the frequency band between 4 and $8 \mathrm{~Hz}$, in time between 130 and $430 \mathrm{~ms}$ from the stimulus onset, and in 7 central electrodes $(3 \mathrm{Z}, 4 \mathrm{Z}, 5 \mathrm{Z}, 4 \mathrm{~L}, 5 \mathrm{~L}, 4 \mathrm{R}, 5 \mathrm{R}$ in the WaveGuard Duke64 system, cf. Appendix Fig. A3 for the locations on the scalp) showed p(FDR-corrected) $<0.05$. The frequency, latency, and scalp distribution matched those in previous AEP studies (Picton \& Hillyard, 1974; Picton et al., 1974). 
AEP amplitude in the tone counting task was computed based on the parameters specified above; EEG data of the tone counting task were band-passed between 4 and $8 \mathrm{~Hz}$. Instantaneous amplitude was computed using the complex-valued analytic signal from the Hilbert transform of the band-passed signal. The amplitude signal was segmented between -500 and 1000 from a tone onset. Each segment was normalized using the pre-stimulus mean and standard deviation. Then the segments were averaged per trial (over 20-24 segments). Using the average waveforms, amplitude values were averaged between 130 and 430ms, and over the 7 central electrodes. This average was used as the AEP amplitude of the trial.

For alpha amplitude, the difference between the resting (pre-task eyes-closed condition) and the task conditions was wide-spread over posterior electrodes consistent with previous studies (Chen et al., 2008; Compton et al., 2019). Of these, we further selected electrodes in which amplitude changed over time. Because we are interested in alpha activity which changes in 2-min interval over tens of minutes, interval series $\pm 30 \mathrm{~min}$ from the intermediate-level $\% \mathrm{MW}$ report was used (reserving the high and low \%MW reports for the main analysis). A linear mixed-effect model, Alpha $\sim$ Time (fixed effect factor) + participants (random effect factor) and its null model, Alpha participants, were fit to the interval series in each of all EEG electrodes (59ch) using the R package, lme4 (Bates, Mächler, Bolker, \& Walker, 2014). Differences between the model fits were tested by ANOVA and chi-squared $\left(\chi^{2}\right)$ statistic. Type-I error over the univariate tests was corrected over the 59 electrodes using the FDR method. Two adjacent right temporal electrodes, 3RB and 3RC (cf. Appendix Fig A3), showed $\chi^{2}(1)=11.23, \mathrm{p}(\mathrm{FDR}$-corrected $)=0.03$, $\eta^{2}=0.43$, power $=0.38$ and $\chi^{2}(1)=12.33, p($ FDR-corrected $)=0.02, \eta^{2}=0.44$, power $=0.34$, respectively. Previous M/EEG studies reported sensor/electrode locations for auditory task-negative alpha activity in the bilateral temporal regions (Lehtelä et al., 1997; Tiihonen et al., 1991). With a threshold of $\mathrm{p}<0.05$, none of the electrodes in the left temporal region qualified. Thus, we averaged alpha amplitude of the two right temporal electrodes and used the result as our measure of DMN activity (Alpha, hereafter).

The trial AEP and Alpha were used for the event time-locked averaging as we described earlier.

\section{Estimation of direction and timing of the neural effects}


A set of linear mixed-effect models were applied to the averaged time series of NoTs, \%CC, AEP and Alpha to obtain $\hat{F}(\Delta t)$ for time lag between 0 and 30 min (Figure 2b). Neural effects from DMN and GDN to NoTs were modeled as;

$$
\text { NoTs } \sim \text { Alpha }_{\Delta t}+\mathrm{AEP}_{\Delta t}+\text { participants }
$$

Alpha and AEP measures enter the linear mixed-effect model as fixed variables. The index, $\Delta t$, is the time lag which changes in 2-min steps, equivalent to 1 bin, between 0 to $30 \mathrm{~min}$. A random variable, participants, was added to take into account individual differences in the data. For the estimation, NoTs data segments between Bins -30 and 30 (shown in the shaded area of Figures $3 a$ and b), and Alpha and AEP segments between Bins (-30- $\Delta t)$ and (30- $\Delta t)$ were used. The length of the segments and the range of the lags were decided based on the available length of the data in this study. This procedure gives $\hat{F}(\Delta t)$ from Alpha to NoTs and that from AEP to in 2-min intervals between 0 and 30min time lags. Statistical significance of the effects was evaluated by comparing (1) to the following reduced models:

$$
\begin{aligned}
& \text { NoTs } \sim \mathrm{AEP}_{\Delta t}+\text { participants } \\
& \text { NoTs } \sim \text { Alpha }_{\Delta t}+\text { participants }
\end{aligned}
$$

Model comparison between (1) and (1.1) uses ANOVA to evaluate the effect of Alpha on NoTs at $\Delta t$, while the comparison between (1) and (1.2) evaluates the effect of AEP on NoTs at $\Delta t$. The chi-squared $\left(\chi^{2}\right)$ was used as the statistic for the model comparison. Type-I error in the ANOVA results over the lags were corrected using the FDR method. Effect size $\left(\eta^{2}\right)$ and power were also checked using R packages esc (Ludecke, 2019) and pwr (Champely, 2020), respectively. The results show when, which neural effect occurred in which direction (positive or negative).

Likewise, models for \%CC were compiled as follows:

$$
\begin{aligned}
& \% \mathrm{CC} \sim \mathrm{AEP}_{\Delta t}+\mathrm{Alpha}_{\Delta t}+\text { participants } \\
& \% \mathrm{CC} \sim \mathrm{Alpha}_{\Delta t}+\text { participants }
\end{aligned}
$$




$$
\% \mathrm{CC} \sim \mathrm{AEP}_{\Delta t}+\text { participants }
$$

Model comparison ( $\chi^{2}$ test) between (2) and (2.1) evaluates the effect of AEP on $\% \mathrm{CC}$ at $\Delta t$, while that between (2) and (2.2) evaluates the effect of Alpha on $\% \mathrm{CC}$ at $\Delta t$. The procedure yields $\hat{F}(\Delta t)$ from Alpha to \% $\mathrm{CC}$ and that from AEP to \% $\mathrm{CC}$ between time lags 0 and $30 \mathrm{~min}$.

The models were fit to the high and low \%MW segments, respectively. In the classical model (Figure 1a), the effect direction is fixed and the time lag is considered negligible. Thus, the effects from Alpha to \%CC and from AEP to NoTs would show the strongest negative effect at $\Delta t=0$, while those from Alpha to NoTs and from AEP to $\% \mathrm{CC}$, would have the strongest positive effect at $\Delta t=0$. In contrast, the current model (Figure 1c) assumes context-dependent change of the function. The effect of Alpha to \% $\mathrm{CC}$ would change from task-negative to positive between the task focused and mind wandering conditions. Moreover, the effects could peak at $\Delta t \neq 0$. 


\section{Results}

\section{Excluded data}

One out of 20 participant was excluded from data analysis due to problems in EEG signal quality. Bad trials, e.g. failure in EEG-marker synchronization, max. 2 trials per participant, were excluded from the analysis. On average, 36.94 good trials/participant were obtained.

\section{Overview}

Figure 3 shows the time courses of \%CC, NoTs, AEP, and Alpha in group average. In the high \%MW segment (Figure $3 \mathrm{a}$ ), Time 0 corresponds to the moment of a $\% \mathrm{MW} \geqq 70$ report. With time, \%CC decreased. The \% $\% \mathrm{CC}$ predicted by the neural measures was the data between Bins -30 and 30 (shaded in gray). The linear trend of the segment was estimated by a mixed-effect model, $\mathrm{Y} \sim$ Time (fixed variable) + participants (random variable). The linear coefficient was $-15.79, \mathrm{t}=-3.80$, and the $95 \%$ confidence interval $(\mathrm{CI})=[-23.66,-7.84]$. Nonlinear patters are also observed, which will report more details later. NoTs first increased, then plateaued about 25 min before the mind wandering. The NoTs plateau was around 3.40 thoughts on average, higher than the 90 percentile of the NoTs distribution in Nakatani et al. (2019). This suggests that at the plateau, the number of thoughts is near the maximum, according to working memory capacity limitations, which is 4 in Luck and Vogel (1997). Consequently, the linear trend between Bins -30 and 30 was modest; Coef. $=0.18, t=1.66$, the 95\% $\mathrm{CI}=[-0.03,0.39]$. As for neural measures, AEP amplitude decreased with time. The linear coefficient was estimated between Bins 30 and 30; Linear coef. $=-0.37, \mathrm{t}=-5.53$, the $95 \% \mathrm{CI}=[-0.50,-0.24]$. The time scale of the decrease is longer than that for adaptation (Pérez-González \& Malmierca, 2014). By contrast, the alpha amplitude increased between Bins -30 and 30; Linear coef. $=0.26, \mathrm{t}=2.59$, the $95 \% \mathrm{CI}=[0.07,0.46]$.

In the low \%MW segments (Figure 3b), \%CC decreased; Linear coef. $=-23.27, \mathrm{t}=-4.97$, the 95\% $\mathrm{CI}=[-32.20,-14.34]$. Also, a local peak was observed around Time 0, the moment of a low \%MW report. 
NoTs slightly increased over time; Linear coef. $=0.55, \mathrm{t}=4.42$, the $95 \% \mathrm{CI}=[0.31,0.78]$. Also, a negative peak was observed at Time 0 . These patterns suggest that the focused state appeared transiently, as participants struggled to keep focus on the simple and repetitive task. The AEP amplitude decreased; Linear coef. $=-0.28, \mathrm{t}=-3.75$, the $95 \% \mathrm{CI}=[-0.43,-0.14]$, while the alpha amplitude increased; Linear coef. $=0.42, \mathrm{t}=3.75$, the $95 \% \mathrm{CI}=[0.210 .64]$. Non-linear patters are also noticeable in the AEP and Alpha.

The linear trends suggest a robust anticorrelation between the spontaneous thought generation and cognitive control, which may be considered as resulting from the time on task. The task was designed to be boring and monotonous, thus to increase spontaneous thoughts and decrease cognitive control with time. Moreover, the linear effect existed both in the high- and low \%MW report conditions. Likewise, the linear components in the neural measures may be considered as resulting from the time. The DMN activity increase while GDN activity decreased with time. The linear neural effects, however, account only for the shift on the antidiagonal in the mental state space.

In contrast, nonlinear neural effects could account for the deviations. Prior to the model fit, we checked if the non-linear patterns in the data are statistically reliable using polynomials. The polynomials showed sizable effects on each measure in both high- and low \% MW segments. The order of the polynomial varied across the measures and segments; the strongest non-linear component was the $27^{\text {th }}$ for $\% \mathrm{CC}$ (coef. $=-10.99, \mathrm{t}=-2.69$, the $95 \% \mathrm{CI}=[18.76,-3.21])$, the $3^{\text {rd }}$ for NoTs $($ coef. $=0.20, \mathrm{t}=1.82$, the $95 \% \mathrm{CI}=[-0.01,0.41])$, the $20^{\text {th }}$ for AEP $($ coef. $=0.13, \mathrm{t}=1.94$, the $95 \% \mathrm{CI}=[0.00,0.25])$, and the $3^{\text {rd }}$ for Alpha (coef. $=0.27, \mathrm{t}=2.61$, the $95 \% \mathrm{CI}=[0.07,0.46])$ in the high $\% \mathrm{MW}$ segment. Those in the low $\% \mathrm{MW}$ segments were; the $17^{\text {th }}$ for $\% \mathrm{CC}($ coef. $=8.59, \mathrm{t}=1.89$, the $95 \% \mathrm{CI}=[17.24,-0.08])$, the $19^{\text {th }}$ for NoTs $($ coef. $=0.26, \mathrm{t}=2.16$, the $95 \% \mathrm{CI}=[0.03,0.50])$, the $10^{\text {th }}$ for AEP $($ coef. $=0.27, \mathrm{t}=3.60$, the $95 \% \mathrm{CI}=[0.13,0.42])$, and the $11^{\text {th }}$ for Alpha (coef. $=-0.18, \mathrm{t}=-1.63$, the $\left.95 \% \mathrm{CI}=[-0.39,0.03]\right)$. Also, multiple higher polynomials showed effects, e.g., the $13^{\text {th }}$ and $27^{\text {th }}$ order of $\% \mathrm{CC}$ in the high $\% \mathrm{MW}$ segment. These results show that nonlinear components in the data were not negligible. At the same time 
the results suggest that the non-linear pattern was too complex to represent with a few selected polynomials.

Figure $3 \mathrm{c}$ combines the measures to plot trajectories between Bins -30 and +30 . In both high- and low $\% \mathrm{MW}$ conditions, the trajectory strayed considerably from the off-diagonal. In the high $\% \mathrm{MW}$ condition, the NoTs were already at high level at Bin-30 while the subsequent trajectory showed a decreasing \%CC (consistent with Nakatani et al, 2019). In the low \%MW condition, the trajectory escaped to the low-NoTs/high-\%CC region, following a complex trajectory. Corresponding neural trajectories also showed complex patterns; in the high \%MW condition, the trajectory moved out of the low-Alpha/highAEP region and dwelled around middle-to-high-Alpha/middle-to-low-AEP regions. In the low \% MW condition, the trajectory dwelled mostly in the middle-to-low-Alpha/middle-to-high-AEP regions in the time period before the low $\% \mathrm{MW}$ report; this may suggest the presence of time lags between the neural and psychological states.

The overview showed that the psychological trajectory deviated from the antidiagonal, and also sizable nonlinear components exist in the corresponding neural activity. Thus, in the following analysis, we tested if the nonlinear neural activity could explain the nonlinear psychological response. As explained in Methods, Estimation of direction and timing of the neural effects, time lags were between 0 and 30min. 
bioRxiv preprint doi: https://doi.org/10.1101/2021.09.04.458977· this version posted September 6, 2021. The copyright holder for this preprint (which was not certified by peer review) is the author/funder, who has granted bioRxiv a license to display the preprint in perpetuity. It is made available under aCC-BY-NC-ND 4.0 International license.

a) HIgh \%MW
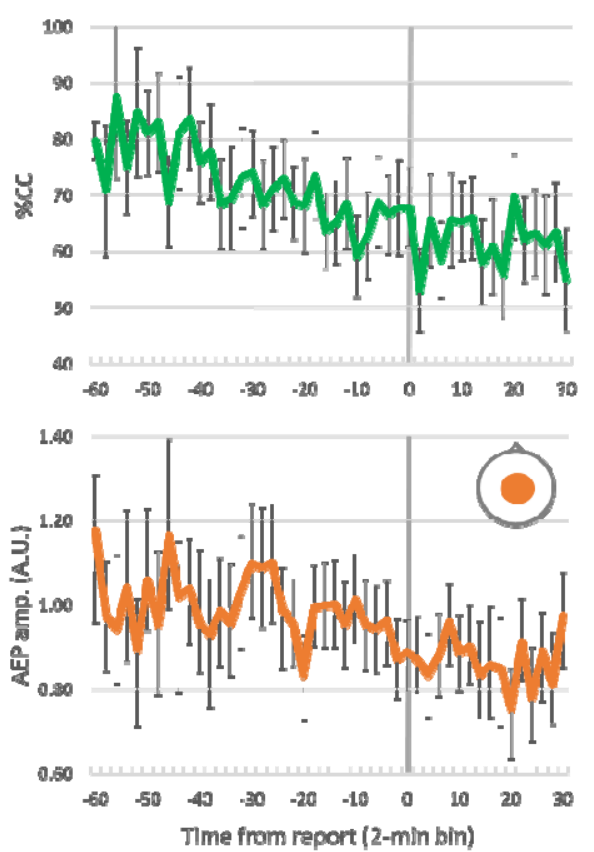

\section{b) Low \%MW}
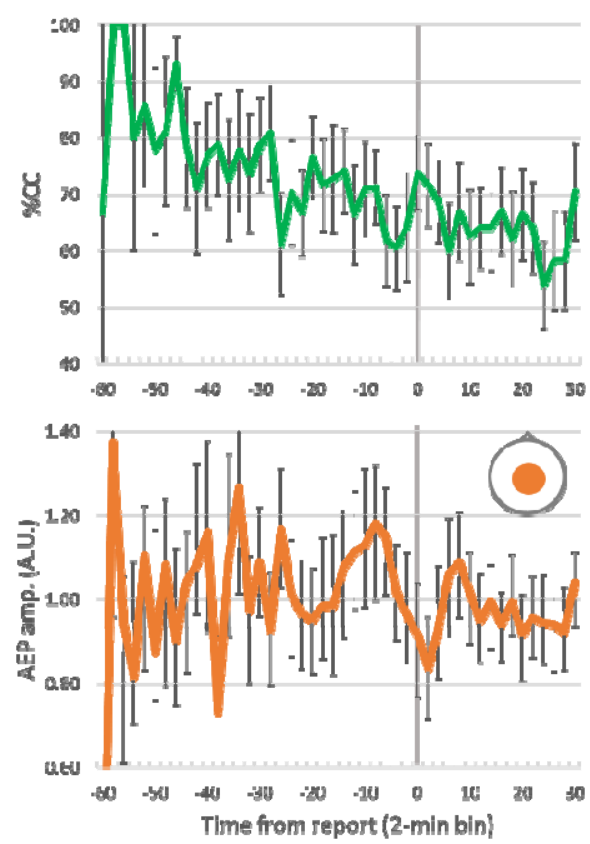
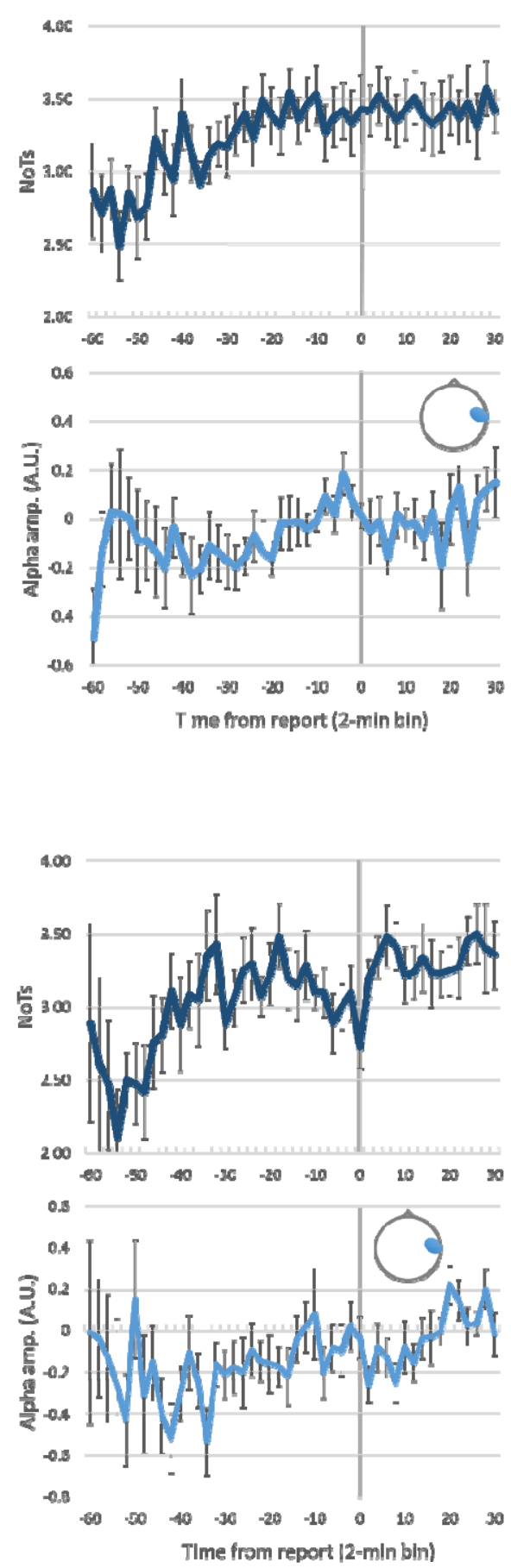
bioRxiv preprint doi: https://doi.org/10.1101/2021.09.04.458977; this version posted September 6, 2021. The copyright holder for this preprint (which was not certified by peer review) is the author/funder, who has granted bioRxiv a license to display the preprint in perpetuity. It is made available under aCC-BY-NC-ND 4.0 International license.

\section{C) Trajectory plots}

\section{High \%MW, \%CC vs. NoTs}

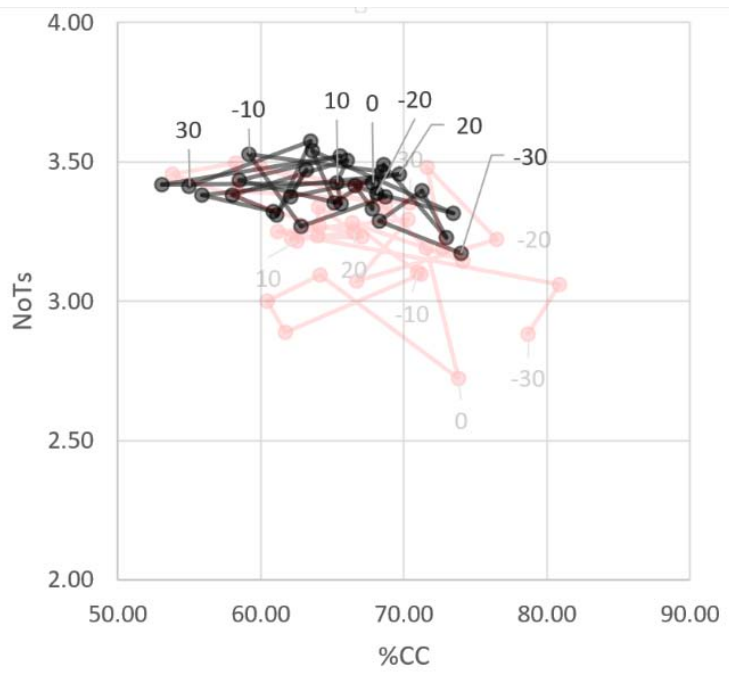

High \%MW, AEP vs. Alpha

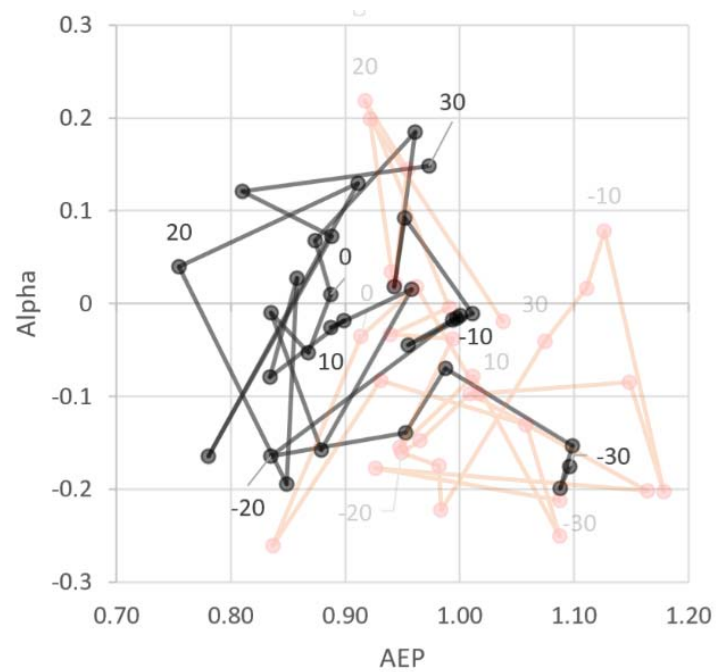

Low \%MW, \%CC vs. NoTs

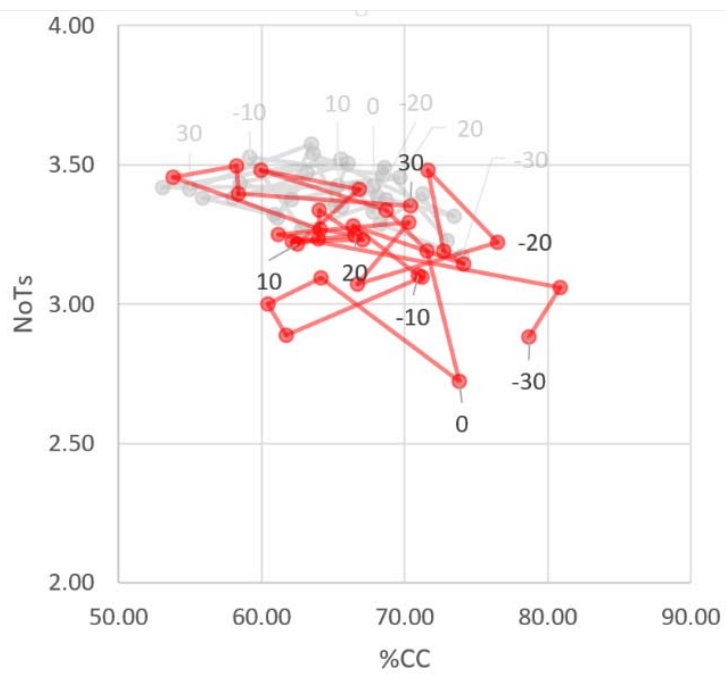

Low \%MW, AEP vs. Alpha

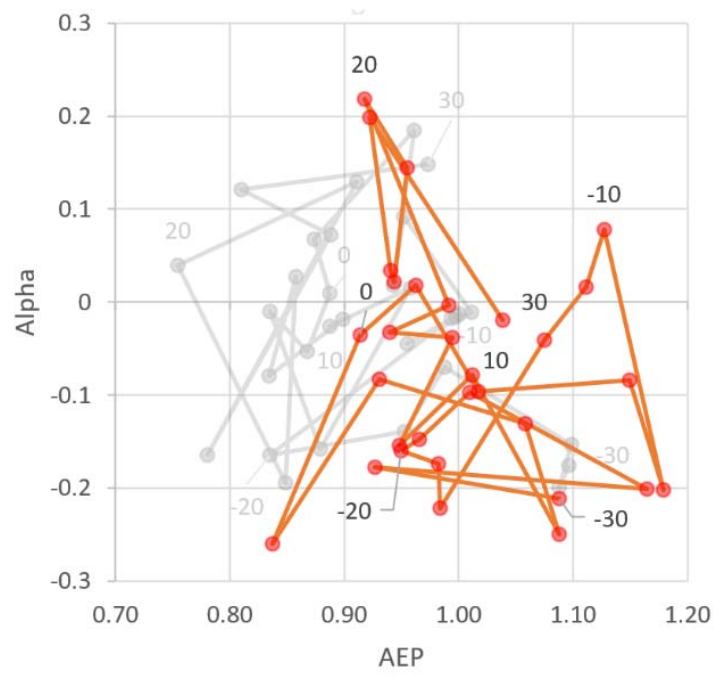


Figure 3. The \%CC, NoTs, AEP, and Alpha amplitudes from high- and low \%MW reports

a) The gray vertical lines indicate the moment of $\% \mathrm{MW} \geqq 70$ report. The $\% \mathrm{CC}$ (green), NoTs (dark blue), AEP (orange) and Alpha (light blue), amplitude are shown in group average. Error bars show Standard Error (SE). Gray shaded segments in the \%CC and NoTs panels are the psychological data to be explained by the models. Head icons in the AEP and Alpha panels indicate electrode locations for the EEG measures. b) The gray vertical lines indicate the timing of the $\% \mathrm{MW}<30$ report. c) Trajectory plots of the measures. Trajectories in the high- (black) and low \% MW (red) conditions were plot between -30 and +30 min period from the moment of the $\% \mathrm{MW}$ report. Digits next to the line graphs indicate the timing in min. Zero indicates the moment of the report. Dimmed line graph of the other condition was added to each panel for visual comparison. 


\section{Timing and direction of neural effects}

Prior to the model fit, the linear trend was removed from the segments of behavioral and neural time series data, to render the $\hat{F}(\Delta t)$ series stationary. This also removed effects which confound with time on task, e.g., fatigue. Figure 4 shows the $\hat{F}(\Delta t)$ of each neural effect. Higher absolute values indicate stronger effects. Four panels in Figure $4 \mathrm{a}$ summarize the results of the high $\% \mathrm{MW}$ segments; The effect from Alpha to NoTs showed a single positive peak at $\Delta t=8 \mathrm{~min}$, while that from Alpha to $\% \mathrm{CC}$ was positive and peaked multiple times between 0 and $24 \mathrm{~min}$. The effect from AEP to NoTs showed peaks between 4 and 14 min. However, the direction of the effect was not consistent. The effect from AEP to \%CC showed positive peaks at 0,8 , and $12 \mathrm{~min}$. The lags were somewhat shorter than those for the alpha amplitude for $\% \mathrm{CC}$.

Low \%MW segments' results are shown in the four panels of Figure 4b. The effect from Alpha to NoTs was positive and peaked at $18 \mathrm{~min}$. The time lag was longer than that in high \%MW segment (peaked at $8 \mathrm{~min}$ ). The effect from Alpha to $\% \mathrm{CC}$ shows a negative peak at $6 \mathrm{~min}$. The direction was opposite to that in high \%MW segments. Panels AEP --> NoTs and AEP --> \%CC, suggest that the effect of AEP was negligible in low \%MW segments.

The results support the current model; The effect of Alpha to \% CC changed task-negative to positive between the task-focused and mind wandering conditions, and time-lagged effects were observed in all routs with possible exception of the rout between AEP and NoTs. Different direction and timing of the neural effects between the conditions suggest that the relationship between the neural activity and psychological functions changed during the tone counting task, which yields complexity to the trajectories between task focused and mind wandering states. 
a) High $\% \mathrm{MW}$
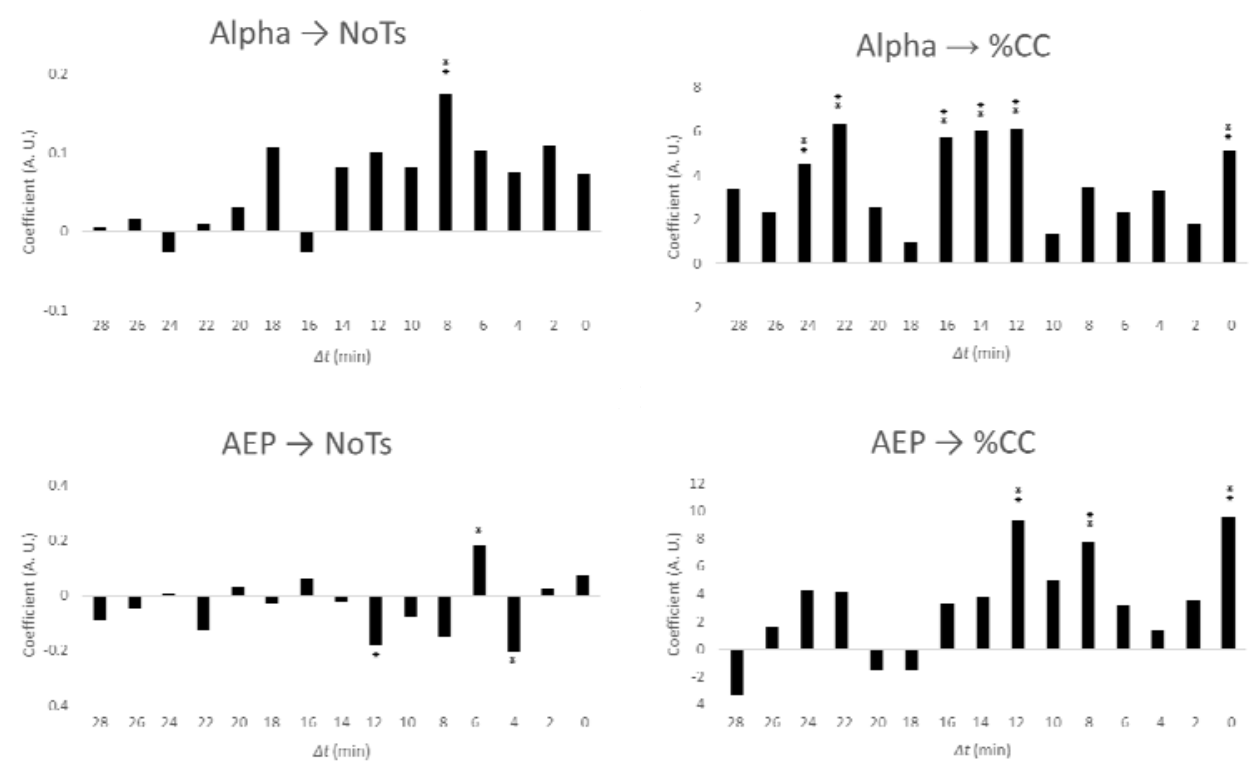

b) Low $\% \mathrm{MW}$
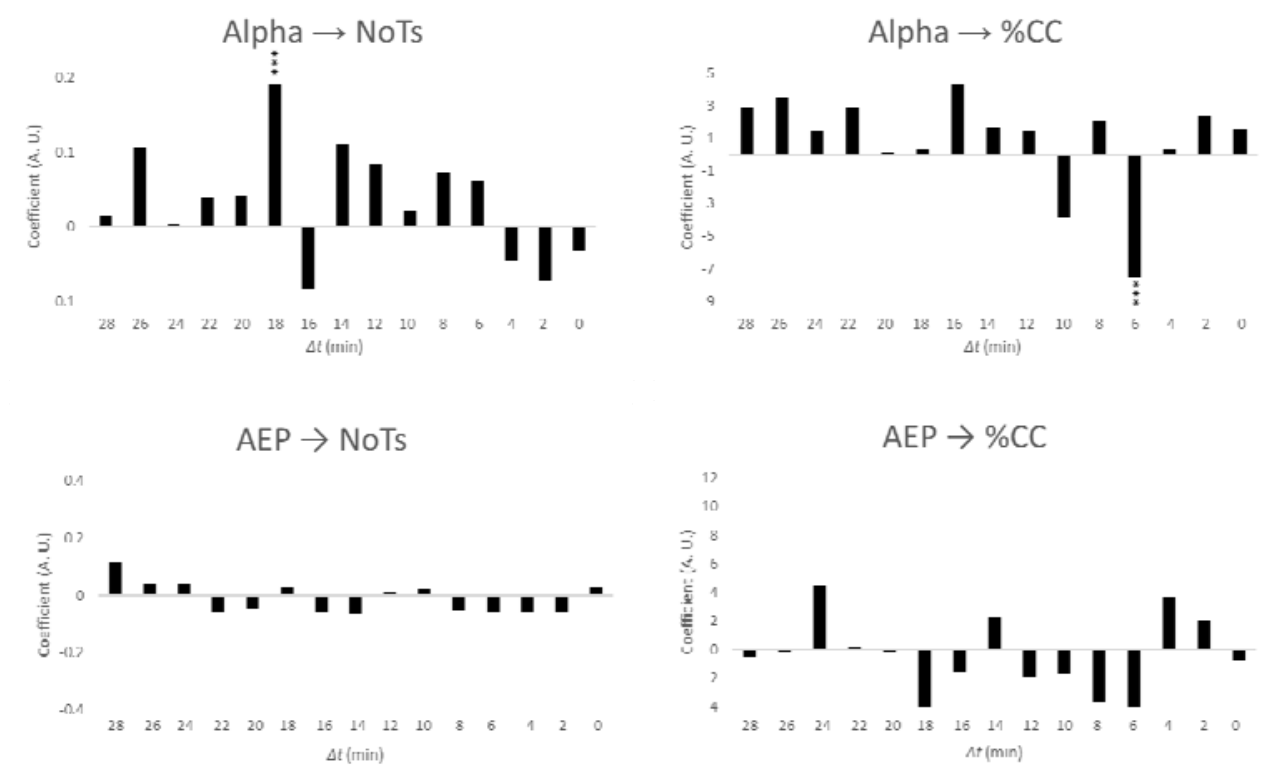
Figure 4. Neural effects at a time lag $(\Delta t)$ between 0 and $30 \mathrm{~min}$.

The $\hat{F}(\Delta t)$ of each neural effects were plot for the high \%MW (a) and low \%MW (b) segments. The estimated coefficients (positive or negative) were plot in from a 30 to $0 \mathrm{~min}$, since a larger $\Delta t$, represents a neural effect in further past. Results of ANOVA $/ \chi^{2}$ test was summarized in asterisk which indicate the probability for the null hypothesis after Type-I error correction by FDR method; *: p < 0.05, **: p < 0.01, and $* * *: p<0.001$. For the lags with $\mathrm{p}<0.01$, effect size $\left(\eta^{2}\right)$ was between 0.39 and 0.48 and the power was between 0.19 and 0.48 . 


\section{Discussion}

Our minds spend significant amounts of time wandering over spontaneous thoughts that do not have immediate relevance to a current task or goal (Antrobus, 1968; Giambra, 1995; Klinger \& Cox, 1987). The trajectory leading from task focused to mind wandering states and back can be complex (Nakatani et al., 2019), and involve itinerancy among a range of other spontaneous mental states (Christoff et al., 2016). The classical neurodynamical model (Figure 1a) which assumes antagonism between the default mode network (DMN) and the goal-directed behavior network (GDN) with fixed effect directions and minimal delays cannot account for the complexity of the trajectory. In the current model (Figure 1c), therefore, we assume that the effect direction and timing are not fixed. The relationship between, on the one hand, DMN and GDN activity and, on the other, spontaneous thought generation and cognitive control was described with dynamic transfer functions. Direction and timing of the neural effects could change with time, or context, i.e. between mind wandering and task focused states. Analyzing EEG data from a tone counting task, we estimated in task focused and mind wandering conditions the direction and timing of the neural effects systematically over the 4 routes of neural effects in the current neurodynamic model

We observed that with time spent on the simple and repetitive task of tone counting, overall, the DMN activity and the number of spontaneous thoughts increased, while GDN activity and task performance decreased. These effects of time on task are, on the one hand, consistent with the idea that anticorrelation in neural effects drives the psychological function. On the other hand, factors such as fatigue and drowsiness could be confounds. Most importantly, the effect of time on task does not explain trajectories deviating from the off-diagonal in Figure 1b. Thus, the time-on-task effect was removed by detrending prior to the estimation of the direction and timing of the neural effects. The classical model predicts the strongest negative effect at $\Delta \mathrm{t}=0$ for from Alpha to $\% \mathrm{CC}$ and from AEP to NoTs, and the strongest positive effect at $\Delta \mathrm{t}=0$ for from Alpha to NoTs and from AEP to \%CC. The results are quite different from these predictions. 
Firstly, DMN effects on cognitive control, which were task-negative in the task focused condition, became task-positive effects about 24 min before a report of mind wandering. This could indicate that participants' mental focus started to shift from external events to internally-motivated thoughts, as suggested by fMRI results in auto-biographical memory studies (Andrews-Hanna et al., 2014; Buckner \& Dinicola, 2019). These studies, however, only reported effects with zero- or short lags, e.g., within $1 \mathrm{~min}$. As we show, the task positive effect peaked repeatedly, with time lags between 0 and 24 mins. This implies that the focused mental state was unstable, sometimes turning inward, for a long period before a report of mind wandering.

DMN activity also showed a positive effect on the generation of spontaneous thoughts. In the mind wandering condition, the effect peaked at a lag of $8 \mathrm{~min}$. The number of thoughts was subjectively reported. Thus, the estimated lags of the DMN effect include delay of report itself. The maximum report delay would be around 2 min since the number was reported after each tone counting trial of which duration was $\sim 2 \mathrm{~min}$. (The report requested every couple of minutes could intrude spontaneousness of the thought generation. It is an interesting question (cf. Hurlburt et al., 2021), but out of scope of the current study.) Even considering the estimation error, the peak was not at lag 0. Taken together, the result implies that a peak of DMN activity increased the thought generation several minutes later.

In the mind wandering condition, the number of the spontaneous thoughts was continuously at a high level, close to four, the limit of working memory (Luck \& Vogel, 1997) This is above the 90\% level of the standardized number of thoughts observed in our previous study (Nakatani et al., 2019). In contrast, the number dropped to 2.72 in the task focused condition, where the DMN effect on thought generation peaked at a lag of $18 \mathrm{~min}$. These results suggest that spontaneous thoughts generated by a critical peak of DMN activity could remain in the memory less than $10 \mathrm{~min}(=18-8)$. After this period, the number drops, allowing the focus to return to the task. The buffer provided by working memory, therefore, could have a critical role in mind wandering and release from it. 
GDN activity showed positive effects on cognitive control with time lags between 0 and 12 mins in the mind wandering condition only. The lagged effects might reflect preparatory activation for the upcoming task-related process. The condition-specific results could be explained by the role of attention in working memory for the tone counting task. Although repetitive and boring, the task is not simple enough to be performed flawlessly without mental effort; the percentage of correct count was around $74 \%$. Crucially, as we have seen, in the mind wandering condition working memory was loaded to nearly full capacity. In this case, selective attention could have a critical role for further processing in the working memory, i.e., the up-dating of the tone count (Bowman \& Wyble, 2007; Simione et al., 2012). By contrast, while the number of thoughts in the task focused condition was below working memory capacity, selective attention may have been less critical. This may explain why in the task focused condition, the sizable fluctuations in auditory EP amplitude did not affect task performance. The absence of GDN effects, together with the above-mentioned salient DMN effects suggest that the focused behavior in the tone counting task was a rebound after the expiry of a period of DMN-initiated elevated thought generation, rather than an intervention of the GDN.

Neural effects with such time lags have hardly been discussed. The present results, however, reveal effects manifesting themselves in behavior with lags of several minutes. This underscores that the assumption of immediate relations between neural activity and psychological functions needs to be scrutinized. Long time-lagged neural effects should be considered as a potential causal factor in the mental dynamics of spontaneous thought processes.

Our study was based on the assumption that activity of DMN and GDN drives spontaneous thought generation and cognitive control (Figure 1). These psychological dimensions are necessary to understand mind wandering in the wider context of spontaneous thinking process; by correlating neural data directly with subjective report of mind wandering, this context would be lost. The dimensionality of the current framework offers a minimum representation of this context, based on the map of spontaneous thinking process by Christoff et al.(2016). To encompass a richer array of spontaneous mental phenomena, 
a higher- dimensional space could be needed (e.g., Voss \& Voss, 2014 uses 3 dimensions to include lucid dream).

The current, minimalistic approach prevents a more specific analyses of the mental states involved in the dimension of cognitive control. Cognitive control is a cluster concept which includes executive control and attention. The functions of cognitive control overlap, at least in part, with the monitoring and control functions that characterize meta cognition (Baars, Ramsøy, \& Laureys, 2003; Flavell, 1979). Meta cognition has been used as an explanatory concept in various descriptions of mind wandering, either explicitly or implicitly. Often after dwelling in a mind wandering state for a while, we realize that we are doing so. This meta cognitive realization is said to play an active role in the restoration of the task focus (Smallwood \& Schooler, 2006). Also, mind wandering could be started intentionally (Seli, Risko, Smilek, \& Schacter, 2016), which likely involves meta cognition. Our focus has been on the neurodynamics that drives the mental state to mind wandering, thereby glossing over the finer distinctions in these mental states, i.e., whether they are intentional or unintentional, cognitive or meta-cognitive. However, we may at least point out that temporal characteristics could be some of the help, e.g., Attention is fast, such as several tens of milliseconds after a stimulus presentation (Picton et al., 1999) and seconds of lapses of attention (Weissman, Roberts, Visscher, \& Woldorff, 2006), while metacognition operates slower, such as several minutes after mind wandering has started. Neural effects observed in different time scale could represent different aspects of the cluster concept for monitor and control functions (Aben et al., 2017; Cohen et al., 1990; Funes et al., 2010; Ridderinkhof, 2002).

Moreover, the current minimalistic approach does not specify individual contributions of DMN and GDN subsystems, such as resting state and saliency networks. Of various subsystems (Cf. Buckner \& Dinicola, 2019 for a recent review), the resting state network of DMN and the attention network of GDN are likely to be reflected in, respectively, the alpha and auditory EP measures of the current study. Other subsystems, such as the saliency network, do not have an established EEG marker. One fMRI study reported contributions of various subsystems to mind wandering (Kucyi, Esterman, Riley, \& Valera, 2016). 
BOLD/fMRI signal from 7 subsystems (3 DMN subsystems: Core DMN, dorsomedial prefrontal cortex, and middle temporal lobule networks and 4 GDN ones: salience, dorsal attention, frontoparietal control, and sensorimotor networks) were correlated with the level of mind wandering (which participants rated in response to probes presented during a sustained visual attention task) and variability of response times (RT) to trials in the 30s intervals before the probes. In all DMN subsystems, the correlation between the BOLD signal and the level of mind wandering was positive, while that between the signal and the RT variance was negative (except for the medial temporal subsystem, which fell short of statistical significance). The latter result indicates that RTs were more stable when the DMN subsystems were highly activated. It, therefore, parallels with our observed task-positive DMN effect. Kucyi et al (2016) found no correlation between the BOLD signal of the GDN subsystems and the level of mind wandering. The authors attribute this to the unreliability of self-report. A positive correlation was found between the signal in the salience and dorsal attention networks and RT variability, which is in accordance with response variability studies (Esterman, Noonan, Rosenberg, \& DeGutis, 2013). These results suggest that, as far as mind wandering is concerned, the respective subsystems of DMN and GDN operate in consort with each other, thereby validating our current minimalistic approach.

Based on the seemingly conflicting nature of the DMN effects (increased mind wandering, yet also increased the response stability), Kucyi et al (2016) concluded that "cognitive processes that spontaneous DMN activity specifically reflects are only partially related to mind-wandering (p. 13899)”. We agree and further propose to evaluate DMN (and GDN) activity in the wider context of spontaneous mental processes. Moreover, we propose to take temporal factors seriously in data analysis, which could separate fluctuation due to the neural dynamics from random noise around mean. We successfully modeled the variability in the psychological measures by past DMN and GDN activity. Temporal order, moreover, underpins the potential causal relationship between the neural activity and psychological functions (Granger, 1969; Pearl, 2000). Based on the current results, we may conclude that DMN activity drives mental state to mind wandering, keeping the number of spontaneous thoughts high and directing the 
mental focus inward, long before mind wandering is reported. DMN activity, moreover, also enables moments of task focus during the boring tone counting task. For the re-establishment of task focus, contribution of GDN activity is negligible, implying cognitive control is not necessary to return to the task-focused state. On the other hand, GDN activity made a positive contribution in the mind wandering state, allowing participants to maintain a basic level of task performance under a high working memory load.

To understand the neurodynamics of mind wandering, a first-order mapping between neural and psychological measures, e.g., correlation between self-report of mind wandering level and alpha band amplitude, is not sufficient. It is also necessary to define a space and time scale in which the dynamics evolve, and the trajectory needs to be studied. The trajectory should be sufficiently long and time-resolved to capture the dynamic characteristics of the process. The time scale of the current study agrees with our subjective experience of mind wandering. Nevertheless, investigation in this time scale has rarely been done. Situated in-between the time scales investigated in previous studies; seconds and shorter with neural measures (Braboszcz \& Delorme, 2011; Ellamil et al., 2016; Kirschner et al., 2012; Kucyi et al., 2016), and hours and longer others with behavioral and subjective report measurements (Bennike, Wieghorst, \& Kirk, 2017; Nakatani et al., 2019), the current study offers a minimal framework for mind wandering and other spontaneous mental processes. 


\section{References}

Aben, B., Verguts, T., \& Van den Bussche, E. (2017). Beyond trial-by-trial adaptation: A quantification of the time scale of cognitive control. Journal of Experimental Psychology: Human Perception and Performance, 43(3), 509-517. doi:10.1037/xhp0000324

Andrews-Hanna, J. R., Saxe, R., \& Yarkoni, T. (2014). Contributions of episodic retrieval and mentalizing to autobiographical thought: Evidence from functional neuroimaging, resting-state connectivity, and fMRI meta-analyses. Neuroimage, 91, 324-335. doi:10.1016/j.neuroimage.2014.01.032

Antrobus, J. S. (1968). Information theory and stimulus - independent thought. British Journal of Psychology, 59(4), 423-430.

Baars, B. J., Rams $\emptyset$ y, T. Z., \& Laureys, S. (2003). Brain, conscious experience and the observing self. Trends in neurosciences, 26(12), 671-675.

Bates, D., Mächler, M., Bolker, B., \& Walker, S. (2014). Fitting linear mixed-effects models using Ime4. arXiv preprint arXiv:1406.5823.

Benjamini, Y., \& Hochberg, Y. (1995). Controlling the false discovery rate: a practical and powerful approach to multiple testing. Journal of the Royal statistical society: series B (Methodological), 57(1), 289-300.

Bennike, I. H., Wieghorst, A., \& Kirk, U. (2017). Online-based Mindfulness Training Reduces Behavioral Markers of Mind Wandering. Journal of Cognitive Enhancement, 1(2), 172-181. doi:10.1007/s41465-017-0020-9

Bernhard, H. (2018). Tracking the mind: measuring the cortical dynamics during mind wandering in an auditory counting task. (Research Master). Maastricht University, Maastricht, The Netherlands.

Bowman, H., \& Wyble, B. (2007). The simultaneous type, serial token model of temporal attention and working memory. 114(1), 38-70. doi:10.1037/0033-295x.114.1.38

Boynton, G. M., Engel, S. A., Glover, G. H., \& Heeger, D. J. (1996). Linear Systems Analysis of Functional Magnetic Resonance Imaging in Human V1. The Journal of Neuroscience, 16(13), 4207-4221. doi:10.1523/jneurosci.16-13-04207.1996

Braboszcz, C., \& Delorme, A. (2011). Lost in thoughts: neural markers of low alertness during mind wandering. Neuroimage, 54(4), 3040-3047.

Brookes, M. J., Hale, J. R., Zumer, J. M., Stevenson, C. M., Francis, S. T., Barnes, G. R., . . Nagarajan, S. S. (2011). Measuring functional connectivity using MEG: methodology and comparison with fcMRI. Neuroimage, 56(3), 1082-1104.

Buckner, R. L., \& Dinicola, L. M. (2019). The brain's default network: updated anatomy, physiology and evolving insights. Nature Reviews Neuroscience, 20(10), 593-608. doi:10.1038/s41583-019-02127

Champely, S. (2020). pwr: Basic Functions for Power Analysis (Version 1.3-0). Retrieved from https://CRAN.R-project.org/package=pwr

Chen, A. C., Feng, W., Zhao, H., Yin, Y., \& Wang, P. (2008). EEG default mode network in the human brain: spectral regional field powers. Neuroimage, 41(2), 561-574.

Christoff, K., Gordon, A. M., Smallwood, J., Smith, R., \& Schooler, J. W. (2009). Experience sampling during $\mathrm{fMRI}$ reveals default network and executive system contributions to mind wandering. Proceedings of the National Academy of Sciences, 106(21), 8719-8724.

Christoff, K., Irving, Z. C., Fox, K. C., Spreng, R. N., \& Andrews-Hanna, J. R. (2016). Mind-wandering as spontaneous thought: a dynamic framework. Nature Reviews Neuroscience, 17(11), 718-731.

Cohen, J. D., Dunbar, K., \& McClelland, J. L. (1990). On the control of automatic processes: A parallel distributed processing account of the Stroop effect. Psychological Review, 97(3), 332-361. doi:10.1037/0033-295x.97.3.332 
Cole, M. W., \& Schneider, W. (2007). The cognitive control network: integrated cortical regions with dissociable functions. Neuroimage, 37(1), 343-360.

Compton, R. J., Gearinger, D., \& Wild, H. (2019). The wandering mind oscillates: EEG alpha power is enhanced during moments of mind-wandering. Cognitive, Affective, \& Behavioral Neuroscience, 19(5), 1184-1191. doi:10.3758/s13415-019-00745-9

Corbetta, M., Kincade, J. M., Ollinger, J. M., McAvoy, M. P., \& Shulman, G. L. (2000). Voluntary orienting is dissociated from target detection in human posterior parietal cortex. Nature Neuroscience, 3(3), 292-297. doi:10.1038/73009

Ellamil, M., Fox, K. C., Dixon, M. L., Pritchard, S., Todd, R. M., Thompson, E., \& Christoff, K. (2016). Dynamics of neural recruitment surrounding the spontaneous arising of thoughts in experienced mindfulness practitioners. Neuroimage, 136, 186-196. doi:10.1016/j.neuroimage.2016.04.034

Esterman, M., Noonan, S. K., Rosenberg, M., \& DeGutis, J. (2013). In the zone or zoning out? Tracking behavioral and neural fluctuations during sustained attention. Cerebral cortex, 23(11), 27122723.

Fink, A., Graif, B., \& Neubauer, A. C. (2009). Brain correlates underlying creative thinking: EEG alpha activity in professional vs. novice dancers. Neuroimage, 46(3), 854-862. doi:10.1016/j.neuroimage.2009.02.036

Flavell, J. H. (1979). Metacognition and cognitive monitoring: A new area of cognitive-developmental inquiry. American Psychologist, 34(10), 906-911. doi:10.1037/0003-066X.34.10.906

Fox, K. C., Spreng, R. N., Ellamil, M., Andrews-Hanna, J. R., \& Christoff, K. (2015). The wandering brain: Meta-analysis of functional neuroimaging studies of mind-wandering and related spontaneous thought processes. Neuroimage, 111, 611-621.

Fox, M. D., Snyder, A. Z., Vincent, J. L., Corbetta, M., Van Essen, D. C., \& Raichle, M. E. (2005). From The Cover: The human brain is intrinsically organized into dynamic, anticorrelated functional networks. Proceedings of the National Academy of Sciences, 102(27), 9673-9678. doi:10.1073/pnas.0504136102

Fransson, P. (2005). Spontaneous low-frequency BOLD signal fluctuations: An fMRI investigation of the resting-state default mode of brain function hypothesis. Human Brain Mapping, 26(1), 15-29. doi:10.1002/hbm.20113

Funes, M. J., Lupiáñez, J., \& Humphreys, G. (2010). Sustained vs. Transient cognitive control: Evidence of a behavioral dissociation. Cognition, 114(3), 338-347. doi:10.1016/j.cognition.2009.10.007

Giambra, L. M. (1995). A laboratory method for investigating influences on switching attention to taskunrelated imagery and thought. Consciousness and cognition, 4(1), 1-21.

Granger, C. W. J. (1969). Investigating Causal Relations by Econometric Models and Cross-spectral Methods. Econometrica, 37(3), 424-438. doi:10.2307/1912791

Greicius, M. D., Krasnow, B., Reiss, A. L., \& Menon, V. (2003). Functional connectivity in the resting brain: A network analysis of the default mode hypothesis. Proceedings of the National Academy of Sciences, 100(1), 253-258. doi:10.1073/pnas.0135058100

Gruberger, M., Simon, E., Levkovitz, Y., Zangen, A., \& Hendler, T. (2011). Towards a Neuroscience of Mind-Wandering. Frontiers in human neuroscience, 5(56). doi:10.3389/fnhum.2011.00056

Hopfinger, J. B., Buonocore, M. H., \& Mangun, G. R. (2000). The neural mechanisms of top-down attentional control. Nature Neuroscience, 3(3), 284-291. doi:10.1038/72999

Hurlburt, R. T., Heavey, C. L., Lapping-Carr, L., Krumm, A. E., Moynihan, S. A., Kaneshiro, C., ... Kelsey, J. M. (2021). Measuring the Frequency of Inner-Experience Characteristics. Perspectives on Psychological Science, 1745691621990379.

Jaarsveld, S., \& Van Leeuwen, C. (2005). Sketches from a Design Process: Creative Cognition Inferred From Intermediate Products. Cognitive science, 29(1), 79-101. doi:10.1207/s15516709cog2901_4 
Jerbi, K., Vidal, J. R., Ossandon, T., Dalal, S. S., Jung, J., Hoffmann, D., . . Lachaux, J.-P. (2010). Exploring the electrophysiological correlates of the default-mode network with intracerebral EEG. Frontiers in Systems Neuroscience. doi:10.3389/fnsys.2010.00027

Kirschner, A., Kam, J. W. Y., Handy, T. C., \& Ward, L. M. (2012). Differential synchronization in default and task-specific networks of the human brain. Frontiers in human neuroscience, 6, 139.

Klinger, E., \& Cox, W. M. (1987). Dimensions of thought flow in everyday life. Imagination, Cognition and Personality, 7(2), 105-128.

Kucyi, A., Esterman, M., Riley, C. S., \& Valera, E. M. (2016). Spontaneous default network activity reflects behavioral variability independent of mind-wandering. Proceedings of the National Academy of Sciences, 113(48), 13899-13904. doi:10.1073/pnas.1611743113

Laufs, H., Krakow, K., Sterzer, P., Eger, E., Beyerle, A., Salek-Haddadi, A., \& Kleinschmidt, A. (2003). Electroencephalographic signatures of attentional and cognitive default modes in spontaneous brain activity fluctuations at rest. Proceedings of the National Academy of Sciences, 100(19), 11053-11058. doi:10.1073/pnas.1831638100

Lehtelä, L., Salmelin, R., \& Hari, R. (1997). Evidence for reactive magnetic $10-\mathrm{Hz}$ rhythm in the human auditory cortex. Neuroscience Letters, 222(2), 111-114. doi:10.1016/s0304-3940(97)13361-4

Luck, S. J., \& Vogel, E. K. (1997). The capacity of visual working memory for features and conjunctions. Nature, 390(6657), 279-281. doi:10.1038/36846

Ludecke, D. (2019). esc: Effect Size Computation for Meta Analysis (Version 0.5.1). Retrieved from https://CRAN.R-project.org/package=esc

Mantini, D., Perrucci, M. G., Del Gratta, C., Romani, G. L., \& Corbetta, M. (2007). Electrophysiological signatures of resting state networks in the human brain. Proceedings of the National Academy of Sciences, 104(32), 13170-13175. doi:10.1073/pnas.0700668104

Marino, M., Arcara, G., Porcaro, C., \& Mantini, D. (2019). Hemodynamic Correlates of Electrophysiological Activity in the Default Mode Network. Frontiers in Neuroscience, 13(1060). doi:10.3389/fnins.2019.01060

Mason, M. F., Norton, M. I., Van Horn, J. D., Wegner, D. M., Grafton, S. T., \& Macrae, C. N. (2007). Wandering minds: the default network and stimulus-independent thought. Science, 315(5810), 393-395.

Menon, V., \& Uddin, L. Q. (2010). Saliency, switching, attention and control: a network model of insula function. Brain Structure and Function, 214(5-6), 655-667.

Nakatani, C., Ganschow, B., \& van Leeuwen, C. (2019). Long-term dynamics of mind wandering: ultradian rhythms in thought generation. Neuroscience of Consciousness, 2019(1). doi:10.1093/nc/niz007

Otto, A. R., Skatova, A., Madlon-Kay, S., \& Daw, N. D. (2015). Cognitive Control Predicts Use of Modelbased Reinforcement Learning. Journal of cognitive neuroscience, 27(2), 319-333. doi:10.1162/jocn_a_00709

Pearl, J. (2000). Causality: Models, reasoning and inference cambridge university press. Cambridge, MA, USA, 9, 10-11.

Pérez-González, D., \& Malmierca, M. (2014). Adaptation in the auditory system: an overview. Frontiers in Integrative Neuroscience, 8(19). doi:10.3389/fnint.2014.00019

Picton, T. W., Alain, C., Woods, D. L., John, M. S., Scherg, M., Valdes-Sosa, P., . . Trujillo, N. J. (1999). Intracerebral Sources of Human Auditory-Evoked Potentials. 4(2), 64-79. doi:10.1159/000013823

Picton, T. W., \& Hillyard, S. A. (1974). Human auditory evoked potentials. II: Effects of attention. Electroencephalography and clinical neurophysiology, 36, 191-200.

Picton, T. W., Hillyard, S. A., Krausz, H. I., \& Galambos, R. (1974). Human auditory evoked potentials. I: Evaluation of components. Electroencephalography and clinical neurophysiology, 36, 179-190.

Pinheiro, J., \& Bates, D. (2006). Mixed-effects models in S and S-PLUS: Springer Science \& Business Media. 
Raichle, M. E. (2015). The Brain's Default Mode Network. Annual Review of Neuroscience, 38(1), 433-447. doi:10.1146/annurev-neuro-071013-014030

Raichle, M. E., Macleod, A. M., Snyder, A. Z., Powers, W. J., Gusnard, D. A., \& Shulman, G. L. (2001). A default mode of brain function. Proceedings of the National Academy of Sciences, 98(2), 676-682. doi:10.1073/pnas.98.2.676

Ridderinkhof, R. (2002). Micro- and macro-adjustments of task set: activation and suppression in conflict tasks. Psychological Research, 66(4), 312-323. doi:10.1007/s00426-002-0104-7

Seeley, W. W., Menon, V., Schatzberg, A. F., Keller, J., Glover, G. H., Kenna, H., . . Greicius, M. D. (2007). Dissociable Intrinsic Connectivity Networks for Salience Processing and Executive Control. Journal of Neuroscience, 27(9), 2349-2356. doi:10.1523/jneurosci.5587-06.2007

Seli, P., Risko, E. F., Smilek, D., \& Schacter, D. L. (2016). Mind-Wandering With and Without Intention. Trends in Cognitive Sciences, 20(8), 605-617. doi:https://doi.org/10.1016/i.tics.2016.05.010

Simione, L., Raffone, A., Wolters, G., Salmas, P., Nakatani, C., Belardinelli, M. O., \& van Leeuwen, C. (2012). "ViSA: A neurodynamic model for visuo-spatial working memory, attentional blink, and conscious access": Correction to Simione et al.(2012).

Smallwood, J., \& Schooler, J. W. (2006). The restless mind. Psychological Bulletin, 132(6), 946-958. doi:10.1037/0033-2909.132.6.946

Tiihonen, J., Hari, R., Kajola, M., Karhu, J., Ahlfors, S., \& Tissari, S. (1991). Magnetoencephalographic 10$\mathrm{Hz}$ rhythm from the human auditory cortex. Neuroscience Letters, 129(2), 303-305. doi:10.1016/0304-3940(91)90486-d

Voss, U., \& Voss, G. (2014). A neurobiological model of lucid dreaming. In R. Hurd \& K. Bulkeley (Eds.), Lucid Dreaming: New Perspectives on Consciousness in Sleep [2 volumes]: New Perspectives on Consciousness in Sleep (Vol. 1, pp. 23-36). Santa Barbara, CA: ABC-CLIO.

Weissman, D. H., Roberts, K. C., Visscher, K. M., \& Woldorff, M. G. (2006). The neural bases of momentary lapses in attention. Nature Neuroscience, 9(7), 971-978. doi:10.1038/nn1727 


\section{Appendix}

\section{Mind wandering percentage (\%MW) histogram}

Figure A1 provides the \%MW histogram over all participants. Low-, intermediate-, and high \%MW trials are marked in yellow $(\% \mathrm{MW}<30)$, gray $(40 \leqq \% \mathrm{MW}<60)$, and purple $(\% \mathrm{MW} \geqq 70)$, respectively

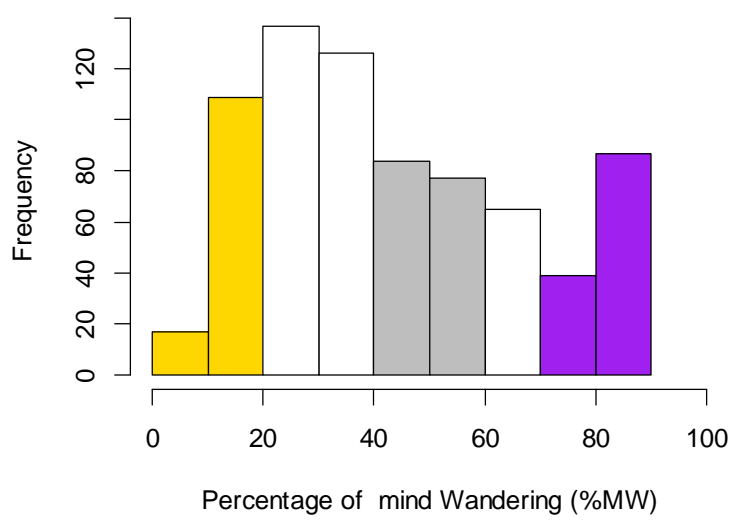

Fig A1. Histogram of the reported mind wandering percentage (\% MW) 


\section{Event time-locked averaging of time series data}

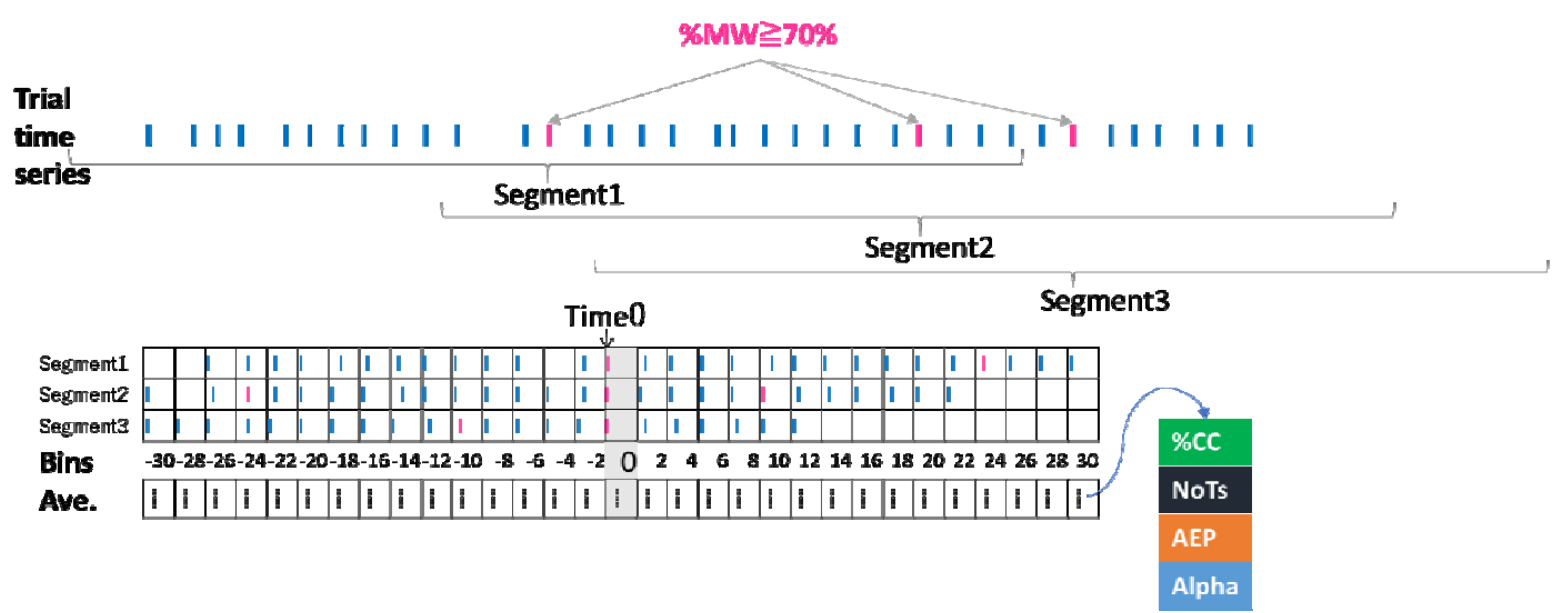

Figure A2. Illustration of event time-locked averaging

In the trial time series (top), trials with high \% MW report ( $\geqq 70 \%$ ) are shown in pink. Segments were defined from the time stamp of the mind wandering percentage report (Time 0 ) between -30 to $+32 \mathrm{~min}$ (middle). The segments were aligned from Time 0, collated in 2-min intervals, and averaged per participant (bottom). Average was made separately for \%CC, NoTs, AEP, and Alpha. Note that bin numbers correspond to the start time of each bin relative to Time 0 .

Table A1. The number bin means

Some participants had bins without a sample mean because the bin had no sample. Table A1 shows the number of the individual bin means (max. 19) for the averaging from the high-, middle-, and low \%MW report, respectively.

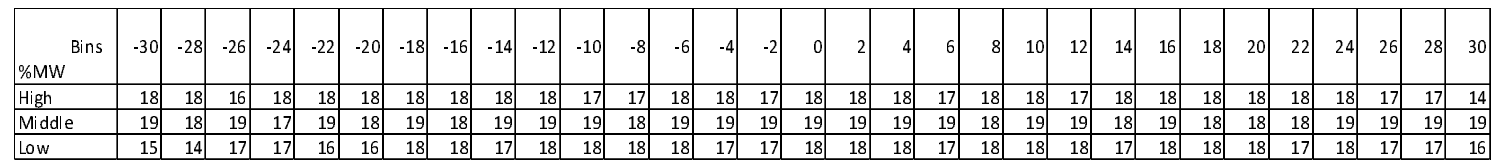




\section{Electrodes for auditory evoked potential (AEP) and Alpha amplitudes}

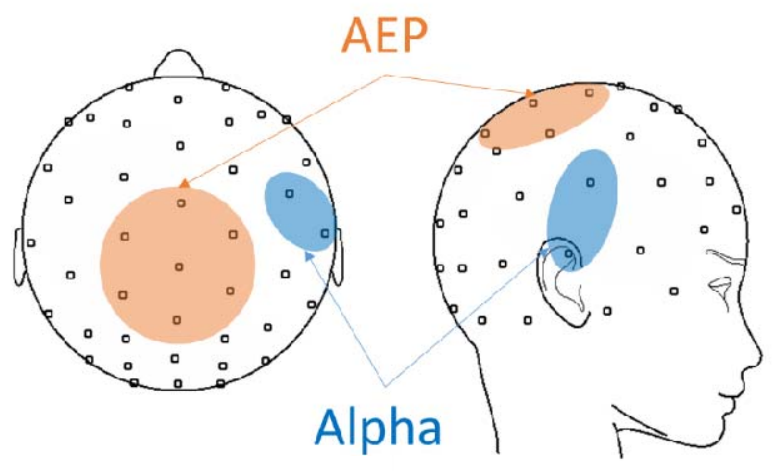

Figure A3. Electrodes for auditory evoked potential (AEP) and Alpha amplitudes

Electrodes used to compute the AEP and Alpha amplitude are indicated. Note that the electrode placement is not the international 10-10, but a manufacturer-defined system. 